\title{
THE RELATIVE BURNSIDE MODULE AND THE STABLE MAPS BETWEEN CLASSIFYING SPACES OF COMPACT LIE GROUPS
}

\author{
NORIHIKO MINAMI
}

Dedicated to Professor Hirosi Toda

\begin{abstract}
Tom Dieck's Burnside ring of compact Lie groups is generalized to the relative case: For any $G \triangleright N$, a compact Lie group and its normal subgroup $A(G \triangleright N)$ is defined to be an appropriate set of the equivalence classes of compact $G$-ENR's with free $N$-action, in such a way that $\psi: A(G \triangleright N) \simeq$ $\pi_{G / N}^{0}\left(S^{0} ; B(N, G)_{+}\right)$, where $B(N, G)$ is the classifying space of principal $(N, G)$-bundle. Under the "product" situation, i.e. $G=F \times K, N=K$, $A(F \times K \triangleright K)$ is also denoted by $A(F, K)$, as it turns out to be the usual $A(F, K)$ when both $F$ and $K$ are finite. Then a couple of applications are given to the study of stable maps between classifying spaces of compact Lie groups: a conceptual proof of Feshbach's double coset formula, and a density theorem on the map $\alpha_{p}^{\wedge}: A(L, H)_{p}^{\wedge} \rightarrow\left\{B L_{+}, B H_{+}\right\}_{p}^{\wedge}$ for any compact Lie groups $L, K$ when $p$ is odd. (Some restriction is applied to $L$ when $p=2$.) This latter result may be regarded as the pushout of Feshbach's density theorem and the theorem of May-Snaith-Zelewski, over the celebrated Carlsson solution of Segal's Burnside ring conjecture.
\end{abstract}

\section{INTRODUCTION}

Even before the arrival of Carlsson's celebrated paper [C], the Segal conjecture for finite groups was known to imply the complete calculation of the stable maps between classifying spaces of finite groups [AGM], [LMM].

More recently, Snaith [S2] got interested in the stable maps between classifying spaces of compact Lie groups, while studying his "Explicit Brauer Induction"problem (see [S2], which is a nice reference on this topic). Snaith's research led to the further generalization of the Segal conjecture by [MSZ]:

Theorem A [MSZ]. Let $F$ and $J$ be finite groups and let $K$ be a compact Lie group. Then $\alpha$ induces the isomorphism

$$
\alpha: A(F \times J, K)_{I(J ; F \times J)}^{\wedge} \rightarrow\left\{B_{F} J_{+}, B_{F} K_{+}\right\}_{F},
$$

where $A(F \times J, K)$ is the Grothendieck $A(F \times J)$-module of principal $(F \times$ $J, K)$-bundles over finite $(F \times J)$-sets, and $(?)_{I(J ; F \times J)}^{\wedge}$ is the completion of

Received by the editors June 23, 1993.

1991 Mathematics Subject Classification. Primary 55P42, 55R35, 57S15; Secondary 55Q91, 55R91.

Key words and phrases. Burnside ring, classifying space, compact Lie groups, stable homotopy theory, Segal conjecture.

This work was partially supported by NSF Grant DMS-8505550 through MSRI. 
$A(F \times J)$-module (?) at the ideal $I(J ; F \times J)$ of elements of $A(F \times J)$ which restrict trivially to $A(H)$ for all $H \subset F \times J$ such that $H \cap J=\{e\}$.

For the notation of the equivariant bundle, see the beginning of $\S 2$. When $F=\{e\}$, this result gives the complete calculation of the stable maps from the classifying space of a finite group to the classifying space of a compact Lie group.

But, as soon as we allow the source to be the classifying space of a general compact Lie group, the situation becomes quite complicated: In the Segal conjecture situation, it has been known that the augmentation ideal does not work [M1] in general. The best positive answer was supplied by M. Feshbach [F2]:

Theorem B (Feshbach's density theorem [F2], [B]). Let $G$ be a compact Lie group. Suppose $T$ is a maximal torus of dimension $n$ and let $W=N_{G} G / T$. Then the map

$$
\alpha: A(G) \rightarrow \pi_{s}^{0}\left(B G_{+}\right)
$$

has a dense image with respect to the skeletal filtration of the target at all odd prime $p$ (i.e. when p-adically completed). Let $\rho: W \rightarrow G l(n, \mathbb{Z})$ be a representation which gives rise to the action of $W$ on $T \approx \mathbb{R}^{n} / \mathbb{Z}^{n}$. Then the Segal conjecture holds at 2 if $\rho$ does not originate at a generalized quaternion group of order $2^{n}, n \geq 4$. In particular the conjecture holds if $n<8$ or if no subquotient of $W$ is isomorphic to the quaternion group of order 16 .

Meanwhile, the surprising paper of S. Bauer [B] found a counterexample to the 2-primary density conjecture (we were told that a preprint version of [F2] suggested a more complicated possible counterexample). Therefore, Feshbach's assumption in Theorem B turned out to be quite essential.

Now the purpose of this paper is to "relativise" tom Dieck's Burnside ring of compact Lie groups and apply it to the study of the stable maps between the classifying spaces of general compact Lie groups:

Let $G \triangleright N$ be a compact Lie group and its normal subgroup. Then

$$
A(G \triangleright N)
$$

is defined to be an appropriate set of the equivalence classes of compact $G$ ENR's with free $N$-action, in such a way that

(1) $A(G \triangleright\{e\})=A(G)$, tom Dieck's Burnside ring of compact Lie group $G[\mathrm{D}]$.

(2) In the "product"case, i.e. $G=L \times K, N=K$, then $A(L \times K \triangleright K)$ is also denoted by $A(L, K)$. This is because, when $F$ is finite, $A(F \times K \triangleright K)$ turns out to be the Grothendieck $A(F)$-module of principal $(F, K)$ bundles over finite $F$-sets, which was denoted by $A(F, K)$ in [MSZ].

$$
\psi: A(G \triangleright N) \simeq \pi_{G / N}^{0}\left(S^{0} ; B(N, G)_{+}\right),
$$

where $B(N, G)$ is the classifying space of principal $(N, G)$-bundle (see $\S 2$ for the definition).

We have a couple of applications of the relative Burnside modules to the study of the stable maps between classifying spaces of compact Lie groups. The first is the compatibility of the pairings:

This result immediately gives a conceptual proof of Feshbach's double coset formula $[\mathrm{F} 1]$ and explains the mysterious similarity between Feshbach's double 
coset formula and the product formula of tom Dieck's Burnside ring of compact Lie groups. Our second application concerns the density problem of the stable maps between the (nonequivariant) classifying spaces of compact Lie groups. In this case, as the skeletal filtration does not give a compact topology in general, the right question to ask is: When $L$ and $K$ are compact Lie groups, does the composite

$$
A(L, H) \stackrel{\alpha}{\rightarrow}\left\{B L_{+}, B H_{+}\right\} \rightarrow \underset{n}{\lim _{n}}\left\{B L_{+}^{(n)}, B H_{+}\right\}_{\text {finite }}^{\wedge}
$$

have a dense image? Here $B L^{(n)}$ is the n-skeleton of $B L$ and $A_{\text {finite }}^{\wedge}=$ $\stackrel{\lim }{\longleftarrow}|A / B|<\infty A / B$ is the profinite completion of an abelian group $A$. Now such a problem can be easily reduced to the $p$-primary cases (see Appendix LocalGlobal), and our main result states

Theorem 3.9. Let $L$ and $H$ be compact Lie groups. Then

$$
\alpha_{p}^{\wedge}: A(L, H)_{p}^{\wedge} \rightarrow\left\{B L_{+}, B H_{+}\right\}_{p}^{\wedge}
$$

has a dense image with respect to the skeletal filtration of the target, if $L$ satisfies the assumption of the Feshbach density theorem, i.e. if either one of the following two conditions holds:

(1) $p$ is odd.

(2) $p=2$ and $\rho: \Pi \rightarrow G l(n, \mathbb{Z})$, given by the Weyl group action on the maximal torus, does not originate at a generalized quaternion group of order $2^{n}, n \geq 4$. More generally, if all the irreducible $\mathbb{Q} \Pi^{\prime}$-summands in the $\Pi^{\prime}$-action on $T^{*} \otimes \mathbb{Q}$ remain irreducible after the 2-adic completion for any 2-subgroup $\Pi^{\prime} \subset W$. In particular, this condition is satisfied, if $n<8$ or if no subquotient of $W$ is isomorphic to the quaternion group of order 16. Here $\Pi$ is the 2-Sylow subgroup of the Weyl group of $L$ and $T$ is a maximal torus of $L$.

Of course, this result can be thought of as a pushout of the aforementioned two theorems of [MSZ] and [F2]. In fact, our proof uses [MSZ] and generalizes the basic idea of [B] (which simplified Feshbach's proof [F2]). But, it should be pointed out that the full generality of the relative Burnside module, i.e. that of non "product"case, was needed to prove Theorem 3.10, which is a "product"case result.

Now, as is shown in the Appendix, Carlsson's affirmative solution of the Segal conjecture [C] and its various generalizations [N], [F2], [MSZ] reduce the density problem of $A(P, K) \rightarrow\left\{B P_{+}, B K_{+}\right\}$to a problem, which involves only relative Burnside modules of $p$-toral (i.e. an extension of a torus by a finite p-group) groups:

Reduction. Let $P$ and $K$ be maximal p-tori of compact Lie groups $L$ and $H$, respectively. Then the map

$$
\alpha_{p}^{\wedge}: A(L, H)_{p}^{\wedge} \rightarrow\left\{B L_{+}, B H_{+}\right\}_{p}^{\wedge}
$$

has a dense image with respect to the skeletal filtration of the target, if

$$
A(P, K)_{p}^{\wedge} \rightarrow \underset{n}{\lim } A\left(F_{n}, K\right)_{p}^{\wedge}
$$


has a dense image, where finite p-subgroups $F_{n}$ 's of $P$ form the following commutative diagram:

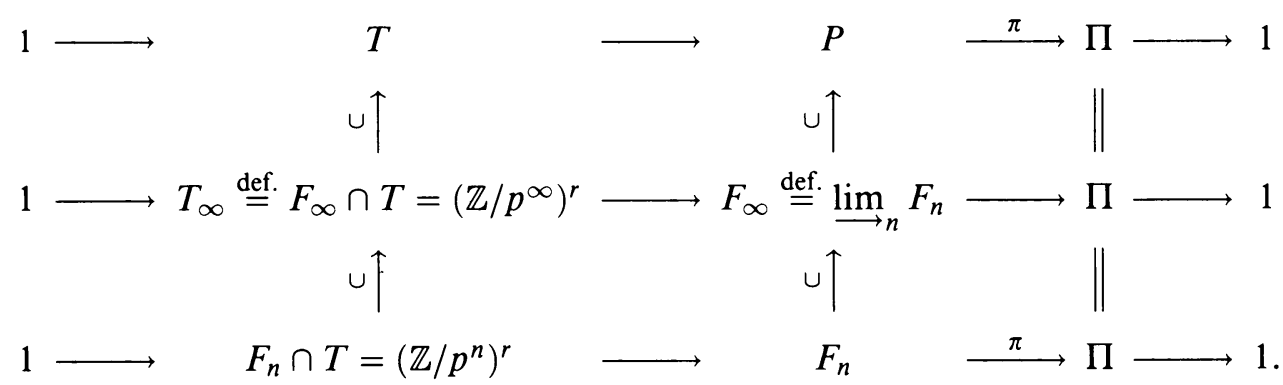

Here $\pi: P \rightarrow \Pi$ is the projection to the finite group of connected components (as $P$ is p-toral $\Pi$ should be a finite p-group), the left-upper map $\left(\mathbb{Z} / p^{\infty}\right)^{r} \rightarrow T$ is the inclusion of the subset consisting of all p-power order elements, and any two such $F_{\infty}$ 's are conjugate to each other in $P$.

This paper is organized as follows:

In $\S 1$, we define $A(G \triangleright N)$ and various functors between the relative Burnside modules.

In $\S 2$, we begin with the isomorphism

$$
\psi: A(L, K) \simeq \pi_{L}^{0}\left(S^{0} ; B_{L} K_{+}\right),
$$

and then we define

$$
\alpha: A(F \times J, K)_{I(J ; F \times J)}^{\wedge} \rightarrow\left\{B_{F} J_{+}, B_{F} K_{+}\right\}_{F}
$$

for general compact Lie groups. Then we state and prove Theorem 2.3, the "universal double coset formula."

In $\S 3$, we prove our main theorem by verifying the condition in the aforementioned Reduction. Our proof strongly depends upon our study of the general (not necessarily "product") relative Burnside modules and greatly influenced by Bauer's [B] simplified proof of Feshbach's density theorem [F2].

In Appendix, we prove Reduction. Here we also show how the profinite density problem (which was stated just before Theorem 3.9. in this introduction) is reduced to the $p$-primary situations.

For more on the related and background material, we refer our survey paper [LM2].

The author would like to express his gratitude to Ralph Cohen for his suggestion to write the survey paper [LM2], which motivated the author to write this up. He also thanks Bill Richter for his generous TeX-nical assistance. He also appreciates Haynes Miller and Stewart Priddy for urging the author to write this paper up. Peter May sent the author lots of preprints which were helpful during the preparation of this paper. The author thanks the referee and Mark Feshbach for carefully reading the preliminary version of this paper and giving him many invaluable suggestions. Finally, the author expresses his gratitude to Masahiro Sugawara for his encouragement.

Notation. Given a map $f: X \rightarrow Y$, its image is denoted by either one of

$$
\operatorname{Im} X=f(X)=\{f(x) \mid x \in X\} .
$$


$G \triangleright N$ and $N \triangleleft G$ means $N$ is a normal subgroup of $G$, and

$$
\begin{aligned}
& N_{G} K=\left\{g \in G \mid g^{-1} K g=K\right\}, \\
& C_{G} K=\{g \in G \mid g k=k g \text { for any } k \in K\},
\end{aligned}
$$

as usual.

When $X$ is a $G$-space, then for any $x \in X$ and a subgroup $H \subseteq G$,

$$
\begin{aligned}
G_{x} & =\{g \mid g \cdot x=x\}, \\
X_{H} & =\left\{x \mid G_{x}=H\right\}, \\
X_{(H)} & =\left\{x \mid G_{x} \text { is conjugate to } H \text { in } G\right\}, \\
X^{H} & =\{x \mid h \cdot x=x \text { for any } h \in H\} .
\end{aligned}
$$

Furthermore,

$$
\{A, B\}_{G}=\pi_{G}^{0}(A, B)
$$

stands for the (abelian) group of $G$ stable homotopy classes from a $G$-spectrum $A$ to a $G$-spectrum $B$.

\section{1. $A(G \triangleright N)$ AND ITS PROPERTIES}

Let $G$ be a compact Lie group and let $N$ be a normal closed subgroup of $G$. Then we define $A(G \triangleright N)$ to be the set of equivalence classes of compact $G$-ENR (for general properties of $G$-ENR, see [J], [Do1], [D2]) with a free $N$-action under the equivalence relation

$$
X \sim Y \Longleftrightarrow \chi\left(X^{S} / N_{N} S\right)=\chi\left(Y^{S} / N_{N} S\right) \quad \text { for any } S \subseteq G,
$$

where $N_{N} S=N_{G} S \cap N$ acts freely on $X^{S}$ and $Y^{S}$ (recall $N$ acts freely on both $X$ and $Y$ ) so that the following diagrams commute:

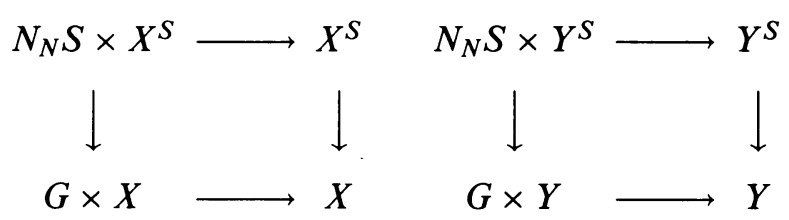

Note that $A(G \triangleright\{e\})=A(G)$, tom Dieck's Burnside ring of compact Lie group $G$ [D]. We define $C^{f}(G \triangleright N)$ to be the set of the conjugacy (by elements of $G$ ) classes of closed subgroups $(H)$ such that $N_{G}(H N) / H N \cong N_{G / N}(H N / N)$ is finite and $H \cap N=\{e\}$. In the "product"case, i.e. when $G=L \times K$ and $N=K$, we write $A(L, K)=A(G \triangleright N)$. Now given $H \subseteq L$ a closed subgroup and $\phi: H \rightarrow K$ a homomorphism, we put $(H, \phi)=\{(m, \phi(m)) \in$ $L \times K \mid m \in H\}$, a closed subgroup of $L \times K$. Then we immediately see that $C^{f}(L \times K \triangleright K)=C^{f}(L, K)$, the set of the conjugacy (by elements of $L \times K$ ) classes of those $(H, \phi)$ such that $\left(N_{L} H\right) / H$ is finite.

We will get a complete description of $A(G \triangleright N)$ (Theorem 1.2). For this purpose we provide an elementary lemma which enables us to deal with Euler characteristic of fixed-point sets and quotient spaces. Here and after, $F \stackrel{i}{\longrightarrow}$ $E \stackrel{p}{\longrightarrow} B$ is said to be a $G$-fiber bundle, if it is a locally $G$-trivial (i.e. locally a product as $G$-spaces) fiber bundle such that both $i$ and $p$ are $G$-equivariant. 
Lemma 1.1.

(i) Let $F \rightarrow E \rightarrow B$ is a $G$-fiber bundle such that a closed normal subgroup $N \triangleleft G$ acts trivially on $B$, then $F^{N} \rightarrow E^{N} \rightarrow B$ is a $G / N$ - fiber bundle.

(ii) Let $F \rightarrow E \rightarrow B$ is a $G$-fiber bundle such that $G$ acts trivially on $B$, then $F / G \rightarrow E / G \rightarrow B$ is a fiber bundle.

Proof. Both can be checked easily using the local triviality.

Theorem 1.2. $A(G \triangleright N)$ is a free abelian group with basis $[G / H]$ for each $(H) \in$ $C^{f}(G \triangleright N)$. For any compact $G$-manifolds $X$ with free $N$-action,

$$
[X]=\sum_{(H)} \chi_{c}\left(X_{(H)} / G\right)[G / H] \in A(G \triangleright N),
$$

where $(H)$ runs over $C^{f}(G \triangleright N)$.

Proof. (See 5.4.4 of [D].) The addition is given by the disjoint union, and the inverse is given by

$$
-[X]=[X \times K],
$$

where $K$ is a compact $G$-ENR with trivial action such that $\chi(K)=-1$. (Note that $X \times K$ is a free $N$ space since $X$ is so.) We want to express any [X], an element of $A(G \triangleright N)$, by elements of the form $[G / H]$ with $(H) \in C^{f}(G \triangleright N)$. We first note that any isotropy type of $X$ is of the desired form $(H)$ such that $H \cap N=\{e\}$. So by the additivity of the Euler-characteristic, we have

$$
\chi\left(X^{S} / N_{N} S\right)=\sum_{(H) \text { such that } H \cap N=\{e\}} \chi_{c}\left(X_{(H)}^{S} / N_{N} S\right),
$$

where $\chi_{c}$ is the Euler characteristic with respect to the homology with compact support. Now recall the fiber bundle:

$$
G / H \rightarrow X_{(H)} \rightarrow X_{(H)} / G .
$$

We apply Lemma 1.1 in two ways: firstly Lemma 1.1(i) for $S \triangleleft N_{G} S \subseteq G$ to the above bundle; secondly Lemma 1.1(ii) for $N_{N} S \subseteq N_{G} S$ to the bundle obtained by the first step. Then, we have the following fiber bundle:

$$
(G / H)^{S} / N_{N} S \rightarrow X_{(H)}^{S} / N_{N} S \rightarrow X_{(H)} / G .
$$

From this, we get

$$
\chi_{c}\left(X_{(H)}^{S} / N_{N} S\right)=\chi\left((G / H)^{S} / N_{N} S\right) \chi_{c}\left(X_{(H)} / G\right) .
$$

Thus, in $A(G \triangleright N)$

$$
[X]=\sum_{(H) \text { such that } H \cap N=\{e\}} \chi_{c}\left(X_{(H)} / G\right)[G / H],
$$

where the summation is over finite terms because (a compact) $G$-ENR has only finitely many orbit types [J]. Now we should prove a couple of claims: (a) $[G / H]=0$ if $N_{G}(H N) / H N$ is not finite; (b) $[G / H]$ 's with $\left(N_{G} H N\right) / H N$ finite are linearly independent. 
For (a), it is sufficient to show $\chi\left((G / H)^{S} / N_{N} S\right)=0$ for any closed subgroup $S \subseteq G$. For this purpose, we study isotropy subgroups of $(G / H)^{S} / N_{N} S$, regarded as a compact $N_{G} H$-ENR under the usual action:

$$
\begin{aligned}
\left(N_{G} H\right) \times\left\{(G / H)^{S} / N_{N} S\right\} & \rightarrow(G / H)^{S} / N_{N} S, \\
\left(n, N_{N} S g H\right) & \mapsto N_{N} S g n^{-1} H .
\end{aligned}
$$

If $n \in N_{G} H$ is contained in the isotropy group at $N_{N} S g H$, we get

$$
\begin{aligned}
& N_{N} S g H=N_{N} S g n^{-1} H \\
& \quad \Longleftrightarrow\left(g^{-1} N_{N} S g\right) H=\left(g^{-1} N_{N} S g\right) n^{-1} H \\
& \quad \Longleftrightarrow n^{-1} \in\left(g^{-1} N_{N} S g\right)(H) .
\end{aligned}
$$

Since $n \in N_{G} H$, this implies that the isotropy group at $N_{N} S g H$ is

$$
\left(g^{-1} N_{N} S g\right)(H) \cap N_{G} H .
$$

Therefore, from the additivity of the Euler characteristic, we only have to show

$$
\chi\left(N_{G} H /\left(g^{-1} N_{N} S g\right) H \cap N_{G} H\right)=0,
$$

for any $g \in G$. Actually, as $\left(g^{-1} N_{N} S g\right) H \cap N_{G} H \subset H N \cap N_{G} H \triangleleft N_{G} H$, it is sufficient to show that the compact Lie group $N_{G} H / N H \cap N_{G} H$ is not finite (then there would be a free $S^{1}$ action on $\left.N_{G} H /\left(g^{-1} N_{N} S g\right) H \cap N_{G} H\right)$. To see this, look at the fiber bundle

$$
N_{G} H / H N \cap N_{G} H \rightarrow N_{G}(H N) / H N \rightarrow N_{G}(H N) /\left(N_{G} H\right)(H N) .
$$

(Note: This is a fiber bundle because $\left(N_{G} H\right)(H N)$ is a subgroup and $N_{G} H / H N$ $\cap N_{G} H \cong\left(N_{G} H\right)(H N) / H N$, for $N_{G} H \subset N_{G}(H N)$. ) Then we immediately find out that the base space $N_{G}(H N) /\left(N_{G} H\right)(H N)$ is finite since $N_{G} H \supset$ $C_{G}(H N)$. On the other hand, the total space $\left(N_{G} H\right)(H N) / H N$ is not finite from the assumption. Therefore the fiber $N_{G} H / N H \cap N_{G} H$ is not finite, as required.

For (b), we first note that

$$
\begin{aligned}
& (G / H)^{H} / N_{N} H=N_{N} H \backslash N_{G} H / H \\
& \quad \cong N_{G} H / H\left(N_{N} H\right)=N_{G} H / H N \cap N_{G} H
\end{aligned}
$$

is finite when $\left(N_{G} H N\right) / H N$ is finite, by the preceding argument for (a). This, in particular, implies that $\chi\left((G / H)^{H} / N_{N} H\right) \neq 0$. Now suppose $[G / H]$ 's, with $N_{G} H / H N$ finite, are linearly dependent and we have a nontrivial linear relation

$$
0=\sum_{(H) \in C^{f}(G \triangleright N)} a_{(H)}[G / H] \in A(G \triangleright N) .
$$

Then, by taking $\left(H^{\prime}\right)$ to be maximal among those such that $a_{(H)} \neq 0$, we get

$$
\begin{aligned}
0 & =\chi\left(\left(\sum a_{(H)}[G / H]\right)^{H^{\prime}} / N_{N} H^{\prime}\right) \\
& \left.=a_{\left(H^{\prime}\right)} \chi\left(G / H^{\prime}\right)^{H^{\prime}} / N_{N} H^{\prime}\right) .
\end{aligned}
$$

However, this is a contradiction, since the last term is nonzero since $a_{\left(H^{\prime}\right)} \neq 0$ and the above remark. This is a contradiction. 
Remark 1.3. For each $(S) \in C^{f}(G \triangleright N)$, we define the homomorphism

$$
\chi^{S}: A(G \triangleright N) \rightarrow \mathbb{Z}
$$

by

$$
[X] \mapsto \chi\left(X^{S} / N_{N} S\right) .
$$

Then these completely characterize $A(G \triangleright N)$ :

$$
A(G \triangleright N) \stackrel{\prod_{(S) \in C^{f}(G \triangleright N)} \chi^{S}}{\longrightarrow} \prod_{S \in C^{f}(G \triangleright N)} \mathbb{Z}
$$

is injective. (Of course, such invariants were used to define $A(G \triangleright N)$, but here we restrict our attention to those $(S) \in C^{f}(G \triangleright N)$.) ) In fact, for any $\sum_{\left(H_{i}\right) \in C^{f}(G \triangleright N)} u_{i}\left[G / H_{i}\right] \in A(G \triangleright N)$, take $H_{j}$ to be maximal among those such that $u_{i} \neq o$. Then

$$
\chi^{H_{j}}\left(\sum u_{i}\left[G / H_{i}\right]\right)=u_{j}\left|N_{G} H_{j} / H_{j} N \cap N_{G} H_{j}\right| \neq 0 \text {. }
$$

Of course, when $N=\{e\}$, this is well known [tD].

Next we define various transformations:

Definition 1.4. (1) Let $G \supset H \supset N$ be a chain of compact Lie groups such that $G \triangleright N$. Then define

$$
\operatorname{Res}_{H}^{G}: A(G \triangleright N) \rightarrow A(H \triangleright N)
$$

by restricting the $G$ action to the $H$ action. When $G=L \times K, H=P \times$ $K, N=K$, this is also denoted by

$$
\operatorname{Res}_{P}^{L}: A(L, K) \rightarrow A(P, K)
$$

for simplicity.

(2) Let $N_{1}$ and $N_{2}$ be closed normal subgroups of $G$ such that $N_{1} \cap N_{2}=$ $\{e\}$. Then the canonical quotient map $\pi: G \rightarrow G / N_{1}$ restricts to the isomorphism $\left.\pi\right|_{N_{2}}: N_{2} \rightarrow N_{2} / N_{2} \cap N_{1} \cong N_{2} N_{1} / N_{1}$. We define

$$
\pi^{*}: A\left(G / N_{1} \triangleright N_{2} N_{1} / N_{1}\right) \rightarrow A\left(G \triangleright N_{2}\right)
$$

by regarding a compact $G / N_{1}$-ENR with a free $N_{2} N_{1} / N_{1}$ action $X$ as a compact $G$-ENR with a free $N_{2}$ action through $\pi$.

(3) Let $N_{1}$ and $N_{2}$ be closed normal subgroup of a compact Lie group $G$ such that $N_{1} \supset N_{2}$. Then define

$$
/ N_{2}: A\left(G \triangleright N_{1}\right) \rightarrow A\left(G / N_{2} \triangleright N_{1} / N_{2}\right)
$$

by $/ N_{2}([C])=\left[X / N_{2}\right]$.

(4) Let $G \triangleright N$ be a compact Lie group and its closed normal subgroup. Let $H$ be a closed subgroup of $G$. Then define

$$
\operatorname{Ind}_{H}^{G}: A(H \triangleright H \cap N) \rightarrow A(G \triangleright N)
$$

by $\operatorname{Ind}_{H}^{G}([X])=\left[G \times{ }_{H} X\right]$. When $G=L \times K, H=P \times K, N=K$, this is also denoted by

for simplicity.

$$
\operatorname{Ind}_{P}^{L}: A(P, K) \rightarrow A(L, K)
$$

(5) Let $N$ be a closed normal subgroup of $G$, which is a closed subgroup of the product of compact Lie groups $G_{1} \times G_{2}$. In other words, $N \triangleleft G \subset G_{1} \times G_{2}$. 
Assume furthermore that $\left.\operatorname{Im} p_{1}\right|_{N} \subseteq N_{1},\left.\operatorname{Ker} p_{1}\right|_{N} \subseteq N_{2}$, where $p_{1}: G_{1} \times G_{2} \rightarrow$ $G_{1}$ is the projection. Then we define the external product

$$
\mathscr{E}: A\left(G_{1} \triangleright N_{1}\right) \otimes A\left(G_{2} \triangleright N_{2}\right) \rightarrow A(G \triangleright N)
$$

by $\mathscr{E}\left(\left[X_{1}\right] \otimes\left[X_{2}\right]\right)=\left[X_{1} \times X_{2}\right]$.

(6) Let $F, K_{1}, K_{2}, K_{3}$ be compact Lie groups. Then define the composition product

$$
\mathscr{C}: A\left(F \times K_{1}, K_{2}\right) \otimes A\left(F \times K_{2}, K_{3}\right) \rightarrow A\left(F \times K_{1}, K_{3}\right)
$$

by $\mathscr{C}\left(\left[X_{1}\right] \times\left[X_{2}\right]\right)=\left[X_{1} \times K_{2} X_{2}\right]$.

Of course, we have to show the well-definedness of these transformations.

(1) Well-definedness of $\operatorname{Res}_{H}^{G}$. This is trivial from our definition.

(2) Well-definedness of $\pi^{*}$. For this, we use Theorem 1.2: We show for any $(H) \in C^{f}\left(G \triangleright N_{2}\right)$ and a compact $G / N_{1}$-ENR with a free $N_{2} N_{1} / N_{1}$ action $X$, when regarded as a compact $G$-ENR, $\chi\left(X_{(H)} / G\right)$ is completely determined by $[X] \in A\left(G / N_{1} \triangleright N_{2} N_{1} / N_{1}\right)$. But this is trivial, because any isotropy subgroup $H$ at an arbitrary point in $X$ contains $N_{1}$ and $X_{(H)} / G=X_{\left(H / N_{1}\right)} /\left(G / N_{1}\right)$ whenever the left side is nonempty.

(3) Well-definedness of $/ N_{2}$. Just as in the proof of the well-definedness of (2), this follows from Theorem 1.2 and an observation that

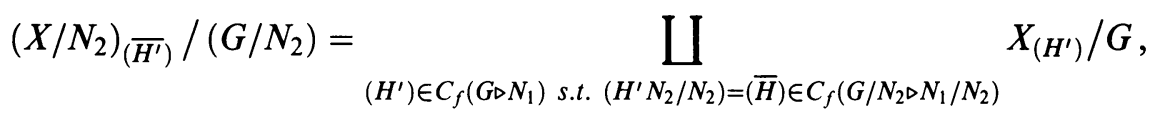

where $X$ is a compact $G$-ENR with a free $N_{1}$-action (so the right-hand side is a finite disjoint union).

To show the well-definedness of $\operatorname{Ind}_{H}^{G}$, we need the following lemma:

Lemma 1.5. Let $G$ be a compact Lie group and let $H$ and $K$ be its closed subgroups. Then there is an isomorphism of finite sets:

$$
(G / H)^{K} / N_{G} K \cong i^{-1}((K)), \quad \text { with } \overline{g H} \mapsto\left(K^{g}\right) \in C(H),
$$

where $i: C(H) \rightarrow C(G)$ is the map between the set of the conjugacy classes of $H$ and $G$, induced by the inclusion $H \subseteq G$.

Proof. $(G / H)^{K} / N_{G} K$ is always finite by p. 87 of [Br]. The isomorphism follows from

$$
\begin{aligned}
\left(K^{g_{1}}\right) & =\left(K^{g_{2}}\right) \in C(H) \\
& \Longleftrightarrow K^{g_{1}}=K^{g_{2} h} \text { for some } h \in H \\
& \Longleftrightarrow N_{G} K g_{1}=N_{G} K g_{2} h \text { for some } h \in H \text { and } g_{1} H \in(G / H)^{K} \\
& \Longleftrightarrow \overline{g_{1} H}=\overline{g_{2} H} \in(G / H)^{K} / N_{G} K,
\end{aligned}
$$

where the second $\Longleftrightarrow$ follows from a sequence of equivalences:

$$
K^{g} \subset H \Leftrightarrow K g H=g H \Leftrightarrow g H \in(G / H)^{K} .
$$

(4) Well-definedness of $\operatorname{Ind}_{H}^{G}$. Let $X$ be a compact $H$-ENR with a free $H \cap N$ action. Then it is easy to see that

$$
\left(G \times_{H} X\right)_{(K)}=\coprod_{\left(K^{\prime}\right) \in i^{-1}((K))} G \times \times_{H}\left(X_{\left(K^{\prime}\right)}\right)
$$


whenever the left side is nonempty, where $i: C(H) \rightarrow C(G)$ is the map between the sets of conjugacy classes induced by the inclusion $H \subseteq G$. From this, we first observe that $G \times{ }_{H} X$ restricts to a free $N$ action if $X$ has a free $H \cap N$ action (since it would imply only those $K^{\prime}$ such that $\{e\}=K^{\prime} \cap H \cap N=K^{\prime} \cap N$ show up). Next, we use this to show that $\chi_{c}\left(\left(G \times_{H} X\right)_{(K)} / G\right)$, with $(K) \in$ $C^{f}(G \triangleright N)$, is completely determined by $[X] \in A(H \triangleright H \cap N)$; this would prove the well-definedness by Theorem 1.2. But, notice that

$$
\begin{aligned}
\chi_{c}\left(\left(G \times_{H} X\right)_{(K)} / G\right) & =\sum_{\left(K^{\prime}\right) \in i^{-1}((K))} \chi_{c}\left(\left(G \times_{H}\left(X_{\left(K^{\prime}\right)}\right)\right) / G\right) \\
& =\sum_{\left(K^{\prime}\right) \in i^{-1}((K))} \chi_{c}\left(X_{\left(K^{\prime}\right)} / H\right),
\end{aligned}
$$

where $\left(K^{\prime}\right) \in C^{f}(H \triangleright H \cap N)$ because $(K) \in C^{f}(G \triangleright N)$ and $N_{H}\left(K^{\prime} H\right) / K^{\prime} N \subseteq$ $N_{G}\left(K^{\prime} H\right) / K^{\prime} N \subseteq N_{G}(K N) / K N$, and we used Lemma 1.5 to guarantee that the sum is a finite sum (or, we could have used the fact that $G \times_{H} X$ is a compact $G$-ENR when $X$ is a compact $H$-ENR). Those $\chi_{c}\left(X_{\left(K^{\prime}\right)} / H\right)$ with $\left(K^{\prime}\right) \in C^{f}(H \triangleright H \cap N)$ can be read off from the basis expression of $[X] \in$ $A(H \triangleright H \cap N))$. This proves the well-definedness.

(5) Well-definedness of $\mathscr{E}$. From the assumption on $N$, we have a short exact sequence

$$
1 \rightarrow N_{N \cap N_{2}} S \rightarrow N_{N} S \rightarrow p_{1}\left(N_{N} S\right) \rightarrow 1
$$

for any closed subgroup $S \subset G$. Then, as $N$ acts freely on $X_{1} \times X_{2}$ for a compact $G_{i}$-ENR $X_{i}$ with a free $N_{i}$ action $(i=1,2)$, we get a fiber bundle

$$
X_{2}^{p_{2}(S)} / N_{N \cap N_{2}} S \rightarrow X_{1}^{p_{1}(S)} \times X_{2}^{p_{2}(S)} / N_{N} S \rightarrow X_{1}^{p_{1}(S)} / p_{1}\left(N_{N} S\right) .
$$

From this, we get

$$
\begin{aligned}
\chi\left(\left(X_{1} \times X_{2}\right)^{S} / N_{N} S\right)= & \chi\left(X_{1}^{p_{1}(S)} / p_{1}\left(N_{N} S\right)\right) \chi\left(X_{2}^{p_{2}(S)} / N_{N \cap N_{2}} S\right) \\
= & \chi\left(X_{1}^{p_{1}(S)} / N_{N_{1}} p_{1}(S)\right) \cdot \chi\left(N_{N_{1}} p_{1}(S) / p_{1}\left(N_{N} S\right)\right) \\
& \cdot \chi\left(X_{2}^{p_{2}(S)} / N_{N_{2}} p_{2}(S)\right) \cdot \chi\left(N_{N_{2}} p_{2}(S) / N_{N \cap N_{2}} S\right),
\end{aligned}
$$

which exhibits the well-definedness of $\mathscr{E}$ by the definition of the relative Burnside module.

(6) Well-definedness of $\mathscr{C}$. Let $\triangle F \subset F \times F$ and $\triangle K_{2} \subset K_{2} \times K_{2}$ be the diagonal subgroups. Then the claim follows from an observation that $\mathscr{C}$ factorize as

$$
\begin{gathered}
A\left(F \times K_{1}, K_{2}\right) \otimes A\left(F \times K_{2}, K_{3}\right) \stackrel{\mathscr{E}}{\rightarrow} A\left(\Delta F \times K_{1}, \Delta K_{2} \times K_{3}\right) \\
\stackrel{\Delta^{\Delta} K_{2}}{\rightarrow} A\left(\Delta F \times K_{1}, K_{3}\right)=A\left(F \times K_{1}, K_{3}\right) .
\end{gathered}
$$

To conclude this section, we record two multiplicative properties of the relative Burnside modules, both of which generalize the multiplicative structure of tom Dieck's Burnside ring. 
Theorem 1.6. (1) $A(G \triangleright N)$ is an $A(G / N)$-module, with respect to the action

$$
\begin{gathered}
\mathscr{A}: A(G / N) \otimes A(G \triangleright N) \rightarrow A(G \triangleright N) \\
{[Y] \otimes[X] \mapsto[Y \times X]}
\end{gathered}
$$

where $Y$ is a compact $G / N-E N R$ and $X$ is a compact $G$-ENR with a free $N$-action.

(2) When $A$ is abelian, $A(L, A)$ becomes an $A(L)$-algebra, with respect to the product

$$
\mathscr{P}: A(L, A) \otimes A(L, A) \rightarrow A(L, A)
$$

by $\mathscr{P}([X] \otimes[Y])=[X \times Y / \sim]$, where $\left(x_{1}, y_{1}\right) \sim\left(x_{2}, y_{2}\right) \Longleftrightarrow \exists a \in$ A s.t. $\left(x_{1}, y_{1}\right)=\left(x_{2} \cdot a, y_{2} \cdot a^{-1}\right)$.

Proof. (1) We only have to check the well-definedness of $\mathscr{A}$. But $\mathscr{A}$ is a special case of $\mathscr{E}$ by embedding $G \subset G \times G \triangleright N$ diagonally. Another approach is to notice

$$
\chi\left((Y \times X)^{S} / N_{N} S\right)=\chi\left(Y^{S N / N}\right) \chi\left(X^{S} / N_{N} S\right)
$$

for any closed subgroup $S \subset G$.

(2) Let $A^{\prime}$ be a left $A \times A$ and free right $A$ action s.t. $\left(a_{1} \times a_{2}\right) \cdot a^{\prime} \cdot a=$ $a_{1} a_{2} a^{\prime} a$. Notice that this is a well-defined action because $A$ is abelian. Then the well-definedness of $\mathscr{P}$ follows from the factorization of $\mathscr{P}$ as

$$
A(L, A) \otimes A(L, A) \stackrel{\mathscr{E}}{\longrightarrow} A(\triangle L, A \times A) \stackrel{\mathscr{C}\left(-\otimes\left[A^{\prime}\right]\right)}{\longrightarrow} A(\Delta L, A),
$$

where the second map is the composition with $\left[A^{\prime}\right] \in A(A \times A, A)$. Then the associativity of $\mathscr{P}$ follows from that of $A$, and the identity element is given by [A], with the trivial left $L$ and the product (free) right $A$ action.

\section{2. $A(G \triangleright N)$ AND THE EQUIVARIANT STABLE HOMOTOPY THEORY}

Let $G \triangleright N$ be a compact Lie group and its normal subgroup as usual. We now recall the classifying space of the equivariant bundles. The general reference would be [LM][Ma].

Let $E(N, G)$ be a universal $N$-free $G$ space; the $G$-homotopy type of $E(N, G)$ is characterized by the requirement that $(E(N, G))^{H}$ be contractible if $H \cap N=\{e\}$ and empty otherwise. We then put $B(N, G)=(E(N, G)) / N$, which is a classifying space of principal $(N, G)$-bundle (which is defined to be the projection of a $N$-free $G$-space to its $N$-orbits). In the "product"case, i.e. when $G=L \times K$ and $N=K$, we write $E_{L} K=E(N, G)$ and $B_{L} K=$ $B(N, G)$. We warn the reader that this notation is different from the more standard notation of the so-called $(\Gamma, G)$-bundle, i.e. $\Gamma$-equivariant $G$-principal bundle [D2]. This corresponds to the product case in our setting, and $B(\Gamma, G)$ $=B_{\Gamma} G$, where $B(\Gamma, G)$ is the notation used in [D2] and not the one used here.

When $A$ is abelian, $B_{L} A$ becomes of Hopf- $L$ space with respect to the $L$-map

$$
B_{L}(m): B_{L} A \times B_{L} A \cong B_{L}(A \times A) \rightarrow B_{L} A,
$$

where the product map $m: A \times A \rightarrow A$ is a homomorphism because $A$ is abelian (see [LMSe]). 
For a given compact $G$-manifold $X$ with a free $N$-action, we have a $G / N$ fiber bundle $X / N \rightarrow$ point, from which we get the transfer stable $G / N$-map

$$
S^{0} \rightarrow X / N_{+} \text {. }
$$

On the other hand, the principal $(N, G)$-bundle $X \rightarrow X / N$ is classified by a $G / N$-map

$$
X / N \rightarrow B(N, G) .
$$

Composing these, we get the stable $G / N$-map

$$
S^{0} \rightarrow B(N, G)_{+} \cdot
$$

We call this $\psi(X)$. We could construct $\phi(X)$ for a general compact $G$-ENR with a free $N$-action $X$, as was shown in [D2], [Do2], [Do3].

Proposition 2.1. $\psi(X) \in \pi_{G / N}^{0}\left(S^{0} ; B(N, G)_{+}\right)$only depends upon $[X] \in$ $A(G \triangleright N)$, and $\phi$ induces the $A(G / N)\left(\cong \pi_{G / N}^{0}\left(S^{0}, S^{0}\right)\right)$-module isomorphism:

$$
\psi: A(G \triangleright N) \cong \pi_{G / N}^{0}\left(S^{0} ; B(N, G)_{+}\right) .
$$

In particular, we have the $A(L)\left(\cong \pi_{L}^{0}\left(S^{0}, S^{0}\right)\right)$-module isomorphism

$$
\psi: A(L, K) \cong \pi_{L}^{0}\left(S^{0} ; B_{L} K_{+}\right)
$$

for the "product"case. Furthermore, when $K$ is abelian, this is an isomorphism of $A(L)$-algebras, where the multiplicative structure of the target is induced from the Hopf- $L$ structure of $B_{L} K$.

Proof. This follows from Theorem 1.2, Theorem 1.6, V.9.3 of [LMS], and Theorem 10 of [LMM].

Now we work with the "product"case and let $L=F \times J$, where $F$ is a finite group. For a given compact $(F \times J) \times K$-manifold $X$ with a free $K$-action, we can define another kind of a stable map. From the $F$-fiber bundle

$$
\left(E_{F} J \times_{J} X\right) / K \simeq E_{F} J \times_{J}(X / K) \rightarrow E_{F} J / J \simeq B_{F} J,
$$

we obtain the transfer stable $F$-map [LMS, IV§3]

$$
B_{F} J_{+} \rightarrow\left(E_{F} J \times{ }_{J} X\right) / K_{+} \text {. }
$$

On the other hand, the principal $(K, K \times F)$-bundle

$$
E_{F} J \times_{J} X \rightarrow\left(E_{F} J \times_{J} X\right) / K
$$

is classified by the classifying $F$-map

$$
\left(E_{F} J \times_{J} X\right) / K \rightarrow B_{F} K .
$$

Now, composing the transfer and the classifying map, we get the $F$-map

$$
\alpha(X): B_{F} J_{+} \rightarrow B_{F} K_{+} .
$$

The significance of $\alpha$ and the relationship between $\alpha$ and $\psi$ are given by the following interpretation: 
Theorem 2.2. The above correspondence factors through $A(F \times J, K)$, and the induced correspondence

$$
\alpha: A(F \times J, K) \rightarrow\left\{B_{F} J_{+}, B_{F} K_{+}\right\}_{F}
$$

can be thought as the composition of $\psi: A(F \times J, K) \simeq \pi_{F \times J}^{0}\left(S^{0} ; B_{F \times J} K_{+}\right)$ and the 0th term of the following composition

$$
\begin{aligned}
\pi_{F \times J}^{*} & \left(S^{0} ; B_{F \times J} K_{+}\right) \rightarrow \pi_{F \times J}^{*}\left(E_{F} J_{+} ; B_{F \times J} K_{+}\right) \\
& \rightarrow \pi_{F \times J}^{*}\left(E_{F} J_{+} ; B_{F} K_{+}\right) \rightarrow \pi_{F}^{*}\left(B_{F} J_{+} ; B_{F} K_{+}\right) .
\end{aligned}
$$

Here the first map is induced by the projection $E_{F} J \rightarrow$ point, the second map is induced by $B_{F \times J} K \simeq B_{\mathfrak{G}^{\prime}} K \rightarrow B_{\mathfrak{G}} K \simeq B_{F} K$, where $\mathfrak{G}^{\prime}$ is the family of closed subgroups $M \subseteq F \times J \times K$ such that $M \cap K=\{e\}, \mathfrak{G}$ is the family of closed subgroups $M \subseteq F \times J \times K$ such that $M J \cap K J=J$, and $B_{\mathfrak{G}^{\prime}} \rightarrow B_{\mathfrak{G}}$ is induced by the inclusion of the families $\mathfrak{G}^{\prime} \rightarrow \mathfrak{G}$. (See the discussion after Theorem 2.3 of [MSZ].)

Proof. This follows from the definitions of $\psi$ and $\alpha$, Proposition 2.1, and the definition of the transfer stable $F$-map [LMS, IV $\S 3$ ] which used the change of universe [LMS,II] argument.

This was originally stated in [MSZ] with respect to their definition of $A(F \times J, K)$, i.e. the Grothendieck $A(F \times J)$-module of principal $(F \times J, K)$ bundle over finite $(F \times J)$-sets. But Theorem 1.2 shows their definition is the same as ours and our definition of $\alpha$ is now shown to generalize that of [MSZ]. In particular, Theorem A in $\S 0$, the main result of [MSZ], is equally valid with respect to our definitions of $A(F \times J, K)$ and $\alpha$.

Theorem 2.3. The paring $\mathscr{C}$ is compatible with the composition of classifying spaces, i.e. we have the following commutative diagram:

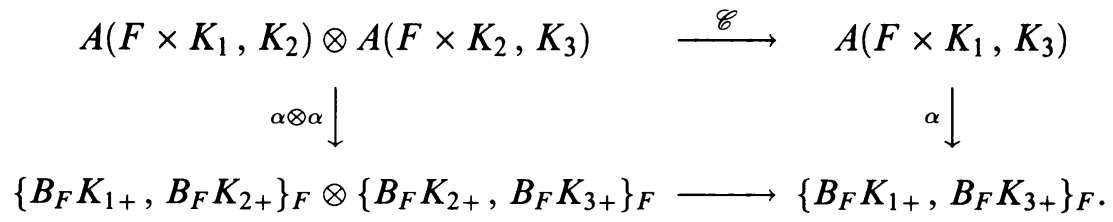

Proof. This compatibility of $\mathscr{C}$ with the composition follows from the following $F \times K_{1}$-diagram (with the pullback square):

$$
\begin{aligned}
& \left.E_{F} K_{1} \underset{K_{1}}{\times}\left(\left(X \underset{K_{2}}{\times} Y\right) / K_{3}\right)\right)=\left(E_{F} K_{1} \underset{K_{1}}{\times} X\right) \underset{K_{2}}{\times}\left(Y / K_{3}\right) \rightarrow E_{F} K_{2} \underset{K_{2}}{\times}\left(Y / K_{3}\right) \rightarrow B_{F} K_{3} \\
& E_{F} K_{1} \underset{K_{1}}{\times}\left(X / K_{2}\right)=\left(E_{F} K_{1} \underset{K_{1}}{\times} X\right) / K_{2} \quad \rightarrow \quad B_{F} K_{2} \\
& \downarrow \\
& B_{F} K_{1} \text {. }
\end{aligned}
$$

Suppose $H$ and $K$ are closed subgroups of $G$; let $\xi(H, G): B_{F} H_{+} \rightarrow$ $B_{F} G_{+}$be the $F$-map induced by the inclusion $H \rightarrow G$, and $\tau(K, G): B_{F} G_{+} \rightarrow$ 
$B_{F} K_{+}$be the stable $F$-transfer induced by the inclusion $K \rightarrow G$. Then Theorem 2.3 conceptually recovers Feshbach's double coset formula (whose equivariant generalization is due to [LMS]), which expresses the composite

$$
\tau(K, G) \xi(H, G): B_{F} H_{+} \rightarrow B_{F} G_{+} \rightarrow B_{F} K_{+} .
$$

Corollary 2.4 ([F1], [LMS]).

$$
\begin{gathered}
\tau(K, G) \xi(H, G) \\
\quad=\sum_{\left(\left(H \cap K^{g}, c_{g^{-1}}\right)\right)} \chi_{c}\left(G_{\left(\left(H \cap K^{g}, c_{g^{-1}}\right)\right)} /(H \times K)\right) c_{g^{-1}} \xi\left(H \cap K^{g}, K^{g}\right) \tau\left(H \cap K^{g}, H\right)
\end{gathered}
$$

where the sum corresponds to the following decomposition:

$$
\begin{aligned}
{[G] } & =\sum_{\left(\left(H \cap K^{8}, c_{g-1}\right)\right)} \chi_{c}\left(G_{\left(\left(H \cap K^{8}, c_{g-1}\right)\right)} /(H \times K)\right)\left[(H \times K) /\left(H \cap K^{g}, c_{g^{-1}}\right)\right] \\
& \in A(H, K),
\end{aligned}
$$

where $G$ is regarded as a compact $H \times K$-ENR with a free $K$-action, by its left $H$ right free $K$-action.

Proof. It is clear that $\xi(H, G)=\alpha([G])$, where this $G$ is considered as a compact $F \times H \times G$-ENR with a free $G$-action, by the trivial $F$-action, the standard left multiplication $H$-action, and the standard right multiplication free $G$-action. Similarly, $\tau(K, G)=\alpha([G])$, where this $G$ is considered as a compact $F \times G \times K$-ENR with a free $K$-action, by the trivial $F$-action, the standard left multiplication $G$-action, and the standard right multiplication free $K$-action.

Then, from Theorem 2. 3 with $K_{1}=H, K_{2}=G, K_{3}=K$, the composition $\tau(K, G) \xi(H, G)$ is given by $\alpha\left(\left[G \times_{G} G\right]\right)$, where $G \times_{G} G$ is regarded as a compact $H \times K$ ENR with a free $K$-action, by the aforementioned left $H$ right $G$ action on the left factor $G$ and the left $G$ right $K$ action on the right factor $G$. But, this is obviously the same as $G$ with the standard left $H$ right $K$ action, as a compact $H \times K$ ENR with a free $K$-action. Therefore, by Theorem 1.2 ,

$$
\begin{aligned}
\tau(K, G) \xi(H, G) & \sum_{\left(\left(H \cap K^{8}, c_{g^{-1}}\right)\right)} \chi_{c}\left(G_{\left(\left(H \cap K^{8}, c_{g^{-1}}\right)\right)} /(H \times K)\right) \alpha\left(\left[(H \times K) /\left(H \cap K^{g}, c_{g^{-1}}\right)\right]\right) .
\end{aligned}
$$

On the other hand, $\alpha\left(\left[(H \times K) /\left(H \cap K^{g}, c_{g-1}\right)\right]\right)$ is easily seen to correspond to the composite of the stable $F$-maps: $B_{F} H_{+} \rightarrow B_{F}\left(H \cap K^{g}\right)_{+} \rightarrow B_{F} K_{+}$, where the first map is the stable $F$-transfer $\tau\left(H, H \cap K^{g}\right)$ and the second map is induced by $c_{g^{-1}}: H \cap K^{g} \rightarrow K$. Of course, this completes the proof.

\section{The DENSITY of $\alpha: A(L, H) \rightarrow\left\{B L_{+}, B H_{+}\right\}$}

In this section, we prove our main theorem. As was explained in the introduction (see Reduction), we only have to show the density of the map between the relative Burnside modules:

$$
A(P, K)_{p}^{\wedge} \rightarrow \underset{\lim }{\longleftarrow} A\left(F_{n}, K\right)_{p}^{\wedge}
$$


We prove this by "relativising" S. Bauer's approach to Feshbach's density theorem as follows:

(I) Construct a subgroup $A_{f}\left(F_{\infty}, K\right)$ of $\lim A\left(F_{n}, K\right)$ generated by elemnts $\left[\left(F_{\infty} \times K\right) /\left(H_{\infty}, \phi\right)\right]$ constructed from each subgroup $H_{\infty}$ of $F_{\infty}\left(=\bigcup_{n} F_{n}\right)$ and a (not necessarily continuous) group homomorphism $\phi: H_{\infty} \rightarrow K$.

(II) Show that $A_{f}\left(F_{\infty}, K\right)$ is dense in $\lim A\left(F_{n}, K\right)_{p}^{\wedge}$.

(III) Show, under our assumption on $P$, that $\left[\left(F_{\infty} \times K\right) /\left(H_{\infty}, \phi\right)\right]$ is "replacable" by elements of the form $\underset{\lim }{\longleftarrow} \operatorname{Res}_{F_{n}}^{P}([(P \times K) /(V, \psi)])$, where $V$ is a closed subgroup of $P$.

Step (I): The definitions of $\left[\left(F_{\infty} \times K\right) /\left(H_{\infty}, \phi\right)\right]$ and $A_{f}\left(F_{\infty}, K\right)$. Let $F_{n}$ be a $p$-group for each $n$, and $F_{\infty}=\cup_{n} F_{n}$ as before. Here we do not necessarily assume that $F_{\infty}$ is a dense subgroup of a $p$-toral subgroup, but we assume that there is a surjective homomorphism $\pi_{F_{\infty}}: F_{\infty} \rightarrow \Pi$, where $\Pi$ is a finite $p$-group such that $\operatorname{Ker} \pi_{F_{\infty}}$ is abelian. We further assume $K$ is $p$-toral and let $\pi_{K}: K \rightarrow \Xi$ be the canonical quotient homomorphism by the normal torus. Then for any subgroup $H_{\infty} \subset F_{\infty}$, the element $\left[\left(F_{\infty} \times K\right) /\left(H_{\infty}, \phi\right)\right]=$ $\prod_{n}\left(x_{F_{n}}\right) \in{\underset{\lim }{\longleftarrow}}_{n} A\left(F_{n}, K\right)$ is defined by

$$
x_{F_{n}}=\lim _{l \rightarrow \infty}\left(\operatorname{Res}_{F_{n}}^{F_{l}}\left[\left(F_{l} \times K\right) /\left(F_{l} \cap H_{\infty},\left.\phi\right|_{F_{l} \cap H_{\infty}}\right)\right]\right) \in A\left(F_{n}, K\right),
$$

where the limit is taken in the $p$-adic completion $A\left(F_{n}, K\right)_{p}^{\wedge}$ (but, still $x_{F_{n}} \in$ $A\left(F_{n}, K\right)$ ). Then $A_{f}\left(F_{\infty}, K\right)$ is defined to be the additive subgroup of $\lim _{n} A\left(F_{n}, K\right)$ generated by all such elements $\left[\left(F_{\infty} \times K\right) /\left(H_{\infty}, \phi\right)\right]$. Of course, we have to justify the definition:

Proposition 3.1. (1) The above element

$$
\left[\left(F_{\infty} \times K\right) /\left(H_{\infty}, \phi\right)\right] \in \underset{n}{\lim } A\left(F_{n}, K\right)
$$

is well-defined.

(2) Suppose $F_{\infty}$ is a dense subgroup of a p-toral $P$ such that $\pi_{F_{\infty}}: F_{\infty} \rightarrow \Pi$ is the restriction of the canonical quotient (by the maximal torus) map $\pi_{P}: P \rightarrow$ $\Pi$. Suppose further that there is a closed subgroup $H$ of $P$ and a continuous homomorphism $\psi: H \rightarrow K$, such that $H \cap F_{\infty}=H_{\infty}$ is dense in $H$ and $\left.\psi\right|_{H_{\infty}}=\phi$. Then

$$
\left[\left(F_{\infty} \times K\right) /\left(H_{\infty}, \phi\right)\right]=\underset{n}{\lim } \operatorname{Res}_{F_{n}}^{P}([(P \times K) /(H, \psi)])
$$

We begin with the key lemma:

Key Lemma. Suppose $H, K$, and $N$ are closed subgroups of a compact Lie groups $G$, such that $N \triangleleft N H=G$ and $K \subset H$. Then we have an isomorphism of compact $K$ manifolds:

$$
\begin{aligned}
& N / H \cap N \rightarrow G / H, \\
& n(H \cap N) \mapsto n H,
\end{aligned}
$$

where $N / H \cap N$ possesses the conjugate $K$ action and $G / H$ possesses the usual left $K$ action:

$$
\begin{aligned}
K \times N / H \cap N & \rightarrow N / H \cap N, & K \times G / H & \rightarrow G / H, \\
(k, n(H \cap N)) & \mapsto k n k^{-1}(H \cap N), & (k, g H) & \mapsto k g H .
\end{aligned}
$$


Proof. This is just an easy verification.

This Key Lemma is most effectively used in the following:

Lemma 3.2. (1) Let $\left\{\Gamma_{n}\right\}_{n \in \mathbb{N}}$ be an increasing sequence of compact Lie groups with a common normal subgroup $\Pi$ such that $\Gamma_{n} / \Pi$ is a finite p-group for any $n \in \mathbb{N}$. Suppose $F$ is a finite p-subgroup of $\Gamma_{\infty}=\bigcup_{n \in \mathbb{N}} \Gamma_{n}$ which intersects trivially with $\Pi$. Then for any closed subgroup $S$ of some $\Gamma_{n}$,

$$
\chi\left(\left(\Gamma_{n} / F\right)^{S} / N_{\Pi} S\right) \in \mathbb{Z}
$$

converges to an integer (not merely to a p-adic integer) as $n$ goes to $\infty$, with respect to the p-adic topology on $\mathbb{Z}$.

(2) Under the situation of (1), suppose that there is a p-toral $\Gamma$, containing $\Gamma_{\infty}$, such that $\Gamma \triangleright \Pi$. Suppose furthermore that the inclusion $\Gamma / \Pi \supset \Gamma_{\infty} / \Pi$ is one of a locally finite p-subgroup in a p-toral, such that any finite p-subgroup of $\Gamma / \Pi$ is conjugate to a subgroup of $\Gamma_{\infty} / \Pi$. Then

$$
\chi\left(\left(\Gamma_{n} / F\right)^{S} / N_{\Pi} S\right) \in \mathbb{Z}
$$

actually converges to

$$
\chi\left((\Gamma / F)^{S} / N_{\Pi} S\right) \in \mathbb{Z}
$$

Proof. (1) Since $F$ is finite, Lemma 1.5 implies that $\left(\Gamma_{\infty} / F\right)^{S}$ has finitely many, say $k, N_{\Gamma_{\infty}} S$-orbits. Therefore, for some $\gamma_{i} \in \Gamma_{\infty}, i=1,2, \ldots, k$, we can write

$$
\begin{aligned}
& \left(\Gamma_{\infty} / F\right)^{S} / N_{\Pi} S=\coprod_{1 \leq i \leq k}\left(N_{\Gamma_{\infty}} S\right) \gamma_{i} F / N_{\Pi} S \\
& \quad=\coprod_{1 \leq i \leq k}\left(N_{\Gamma_{\infty}} S /\left(F_{i}^{\gamma_{i}^{-1}} \cap N_{\Gamma_{\infty}} S\right)\right) / N_{\Pi} S \\
& =\coprod_{1 \leq i \leq k}\left(N_{\Gamma_{\infty}} S / N_{\Pi} S\right) /\left(N_{\Pi} S\left(F^{\gamma_{i}^{-1}} \cap N_{\Gamma_{\infty}} S\right) / N_{\Pi} S\right)\left(\because N_{\Pi} S \triangleleft N_{\Gamma_{\infty}} S\right) \\
& =\coprod_{1 \leq i \leq k} \underset{n}{\lim }\left(N_{\Gamma_{n}} S / N_{\Pi} S\right) /\left(N_{\Pi} S\left(F^{\gamma_{i}^{-1}} \cap N_{\Gamma_{n}} S\right) / N_{\Pi} S\right) .
\end{aligned}
$$

Note that

$$
N_{\Gamma_{\infty}} S / N_{\Pi} S \quad\left(\subset \Gamma_{\infty} / \Pi\right)
$$

is a locally finite $p$-group and $N_{\Pi} S\left(F^{\gamma_{i}^{-1}} \cap N_{\Gamma_{\infty}} S\right) / N_{\Pi} S$ is its subgroup. Therefore, if

$$
\left(N_{\Gamma_{\infty}} S / N_{\Pi} S\right) /\left(N_{\Pi} S\left(F^{\gamma_{i}^{-1}} \cap N_{\Gamma_{\infty}} S\right) / N_{\Pi} S\right)
$$

is infinite,

$$
\underset{n}{\lim }\left|\left(N_{\Gamma_{n}} S / N_{\Pi} S\right) /\left(N_{\Pi} S\left(F^{\gamma_{i}^{-1}} \cap N_{\Gamma_{n}} S\right) / N_{\Pi} S\right)\right|=0 \in \mathbb{Z}
$$

with respect to the $p$-adic topology on $\mathbb{Z}$. This implies that

$$
\chi\left(\left(\Gamma_{n} / F\right)^{S} / N_{\Pi} S\right)=\left|\left(\Gamma_{n} / F\right)^{S} / N_{\Pi} S\right|
$$


converges to the sum of those

$$
\left|\left(N_{\Gamma_{\infty}} S / N_{\Pi} S\right) /\left(N_{\Pi} S\left(F^{\gamma_{i}^{-1}} \cap N_{\Gamma_{\infty}} S\right) / N_{\Pi} S\right)\right|
$$

which are finite. Of course, such a sum is a natural number.

(2) As $F$ is a finite $p$-group, we may assume that $S$ is also a finite $p$-group without any loss of generality. We first prove that the natural map

$$
\left(\Gamma_{\infty} / F\right)^{S} / N_{\Gamma_{\infty}} S \rightarrow(\Gamma / F)^{S} / N_{\Gamma} S
$$

is an isomorphism. Applying Lemma 1.5 repeatedly, we see it is enough to prove that $\left(\Gamma / \Gamma_{\infty}\right)^{S} / N_{\Gamma} S$ consists of a single element. For this, let $T$ be the maximal torus of the $p$-toral $\Gamma$ and $T_{\infty}=T \cap \Gamma_{\infty}$. Then the Key Lemma says that

$$
\left(\Gamma / \Gamma_{\infty}\right)^{S} \cong\left(T / T_{\infty}\right)^{S}
$$

where the $S$-action on $\left(T / T_{\infty}\right)$ is the conjugate action. This right-hand side shows up in the cohomology long exact sequence:

$$
0 \rightarrow\left(T_{\infty}\right)^{S} \rightarrow T^{S} \rightarrow\left(T / T_{\infty}\right)^{S} \stackrel{\delta}{\longrightarrow} H^{1}\left(S, T_{\infty}\right) \rightarrow \cdots
$$

where $\delta$ is the connecting homomorphism. However, $\delta$ is a null homomorphism, because $\left(T / T_{\infty}\right)^{S}$ is a $\mathbb{Z}\left[\frac{1}{p}\right]$-module and $H^{1}\left(S, T_{\infty}\right)$ is $|S|$-torsion (recall that $S$ is a finite $p$-group). Therefore, we find that

$$
T^{S} \rightarrow\left(T / T_{\infty}\right)^{S}
$$

is an epimorphism. But, this fact and the Key Lemma (and its proof) implies that $\left(\Gamma / \Gamma_{\infty}\right)^{S}$ consists of a single $C_{T} S$ orbit. As $C_{T} S \subset N_{\Gamma} S$, this proves that $\left(\Gamma / \Gamma_{\infty}\right)^{S} / N_{\Gamma} S$ consists of a single element.

The preceding argument implies that, using $\gamma_{i} \in \Gamma_{\infty}(i=1,2, \ldots, k)$ in (1) again, we can also write

$$
(\Gamma / F)^{S} / N_{\Pi} S=\coprod_{1 \leq i \leq k}\left(N_{\Gamma} S / N_{\Pi} S\right) /\left(N_{\Pi} S\left(F^{\gamma_{i}^{-1}} \cap N_{\Gamma} S\right) / N_{\Pi} S\right) .
$$

Therefore, to prove

$$
\underset{n}{\lim } \chi\left(\left(\Gamma_{n} / F\right)^{S} / N_{\Pi} S\right)=\chi\left((\Gamma / F)^{S} / N_{\Pi} S\right)
$$

it suffices to show, for each $i$,

$$
\begin{aligned}
& \underset{n}{\lim } \chi\left(\left(N_{\Gamma_{n}} S / N_{\Pi} S\right) /\left(N_{\Pi} S\left(F^{\gamma_{i}^{-1}} \cap N_{\Gamma_{n}} S\right) / N_{\Pi} S\right)\right) \\
&=\chi\left(\left(N_{\Gamma} S / N_{\Pi} S\right) /\left(N_{\Pi} S\left(F^{\gamma_{i}^{-1}} \cap N_{\Gamma} S\right) / N_{\Pi} S\right)\right),
\end{aligned}
$$

where the limit is taken with respect to the $p$-adic topology. Now, to prove this, it suffices to show that $N_{\Gamma} S$ is a $p$-toral (then, it easily follows that

$$
\begin{aligned}
& \left(N_{\Gamma_{\infty}} S / N_{\Pi} S\right) /\left(N_{\Pi} S\left(F^{\gamma_{i}^{-1}} \cap N_{\Gamma_{\infty}} S\right) / N_{\Pi} S\right) \\
& \subseteq\left(N_{\Gamma} S / N_{\Pi} S\right) /\left(N_{\Pi} S\left(F^{\gamma_{i}^{-1}} \cap N_{\Gamma} S\right) / N_{\Pi} S\right)
\end{aligned}
$$


is a dense locally finite $p$-subgroup of a $p$-toral and then the claim about Euler characteristics can be shown as in (1)).

To prove $N_{\Gamma} S$ is a $p$-toral, we only have to show that $N_{(S T)} S / S=(S T / S)^{S}$ is a $p$-toral. Now the Key Lemma says this is nothing but $(T / S \cap T)^{S}$, where the $S$ action on $(T / S \cap T)$ is a conjugate one. But such a conjugate invariant of a torus $(T / S \cap T)=T^{n}$, say, by a finite $p$-group $S$ is is easily seen to be a $p$-toral. In fact, from the covering homotopy property, there is a short exact sequence of $S$ modules $0 \rightarrow \mathbb{Z}^{n} \rightarrow \mathbb{R}^{n} \rightarrow T^{n} \rightarrow 0$. Then, from the associated cohomology long exact sequence, we get the following exact sequence:

$$
0 \rightarrow\left(\mathbb{R}^{n}\right)^{S} /\left(\mathbb{Z}^{n}\right)^{S} \rightarrow\left(T^{n}\right)^{S} \rightarrow H^{1}\left(S, \mathbb{Z}^{n}\right),
$$

where $H^{1}\left(S, \mathbb{Z}^{n}\right)$ is an abelian $p$-group, by the transfer argument, and $\left(\mathbb{R}^{n}\right)^{S} /\left(\mathbb{Z}^{n}\right)^{S}$ is a torus. Of course, we could have used the Tate cohomology to avoid using the covering homotopy property.

Proof of Proposition 3.1(1). To show the well-definedness of $\left[\left(F_{\infty} \times K\right) /\left(H_{\infty}, \phi\right)\right]$ we prepare the following: Let $G_{\infty}$ be the subgroup of $F_{\infty} \times K$, generated by $\left(H_{\infty}, \phi\right)$ and $\operatorname{Ker} \pi_{F_{\infty}} \times \operatorname{Ker} \pi_{K}$. Let

$$
N_{\infty}=H_{\infty} \cap \operatorname{Ker} \pi_{F_{\infty}} \cap \phi^{-1}\left(\operatorname{Ker} \pi_{K}\right),
$$

then $N_{\infty} \triangleleft H_{\infty}$ with a finite $p$-group quotient

$$
H_{\infty} / N_{\infty} \underset{\Re F_{\infty} \times \pi_{K}}{\cong} \pi_{F_{\infty}}\left(H_{\infty}\right) \times \pi_{K}\left(H_{\infty}\right) \cong \Pi \times \Xi .
$$

It should be noted that $\overline{\left(H_{\infty}, \phi\right)} \stackrel{\text { def }}{=}\left(H_{\infty}, \phi\right) /\left(N_{\infty},\left.\phi\right|_{N_{\infty}}\right) \cong H_{\infty} / N_{\infty}$ is a finite $p$-group, rather than infinite, and is a normal subgroup of $G_{\infty}$ that intersects trivially with $K$.

Then consider the following system of groups and group homomorphisms:

$$
\begin{aligned}
& G_{n}=G_{\infty} \cap\left(F_{n} \times K\right), \\
& N_{n}=N_{\infty} \cap F_{n}, \\
& \bar{G}_{n}=G_{n} /\left(N_{n},\left.\phi\right|_{N_{n}}\right), \\
& K^{\prime}=K \cap G_{\infty}, \\
& \rho_{n}: G_{n} \rightarrow \bar{G}_{n}, \text { the canonical projection, } \\
& \overline{K^{\prime}}=\rho_{\infty}\left(K^{\prime}\right) \subseteq \bar{G}_{\infty} .
\end{aligned}
$$

Since $\operatorname{Ker} \pi_{K}=K \cap \operatorname{Ker} \pi_{K} \subseteq K \cap G_{n} \subseteq K$ and $K / \operatorname{Ker} \pi_{K}=\Xi$ is finite, $K^{\prime}=K \cap G_{n}$ for any sufficiently large $n$. For such $n, \overline{G_{n}}$ contains $\overline{K^{\prime}}\left(\cong K^{\prime}\right)$ as a matter of course. With these data, Definition 1.4 enables us to define

$$
\begin{aligned}
\rho_{n}^{*}: A\left(\bar{G}_{n} \triangleright \overline{K^{\prime}}\right) & \rightarrow A\left(G_{n} \triangleright K^{\prime}\right), \\
\operatorname{Ind}_{G_{n}}^{F_{n} \times K}: A\left(G_{n} \triangleright K^{\prime}\right) & \rightarrow A\left(F_{n} \times K \triangleright K\right) .
\end{aligned}
$$


In fact, these form maps between systems of the relative Burnside modules:

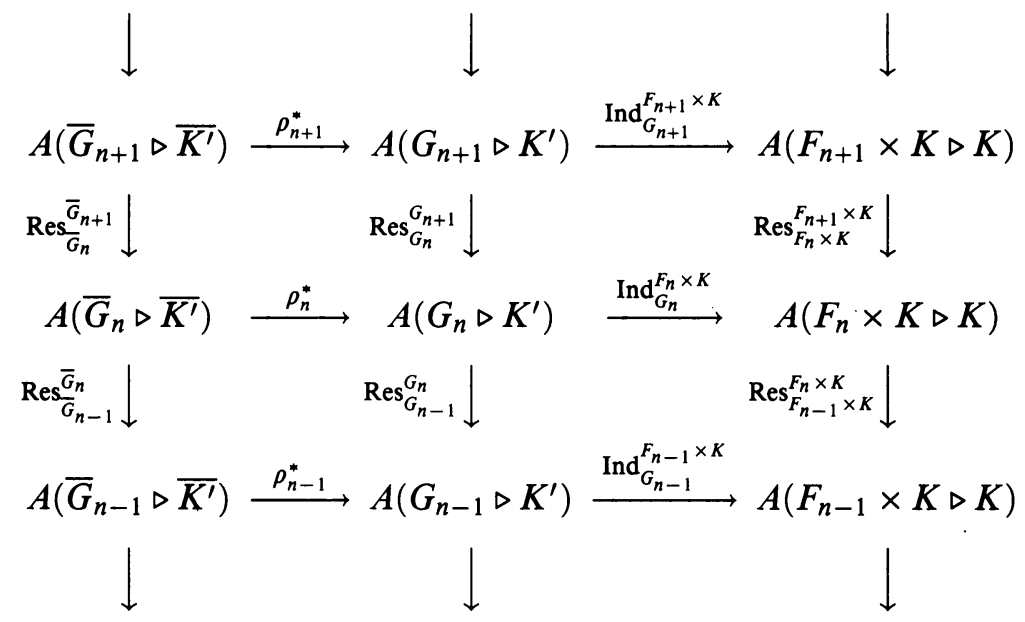

Here the commutativities of the left side squares are trivial to see. But, to guarantee the commutativities of the right side squares, we needed $n$ to be sufficiently large so that $\left(F_{n+1} \times K\right) / G_{n+1} \cong\left(F_{n} \times K\right) / G_{n}$. The reason why $\left(F_{n} \times K\right) / G_{n}$ stabilizes can be seen from the diagram:

$$
\begin{aligned}
\left(F_{n} \times K\right) / G_{n} & =F_{n} \times K /\left(F_{n} \times K\right) \cap G_{\infty} \subseteq F_{\infty} \times K / G_{\infty} \\
& \leftarrow F_{\infty} \times K / \operatorname{Ker} \pi_{F_{\infty}} \times \operatorname{Ker} \pi_{K} \cong \Pi \times \Xi, \text { a finite group }
\end{aligned}
$$

More precisely, $\left(F_{n} \times K\right) / G_{n}$ is non-decreasing and its limit $F_{\infty} \times K / G_{\infty}$ is finite, and so it should stabilize after sufficiently large $n$.

Now the point is

$$
\left[\left(F_{n} \times K\right) /\left(H_{n},\left.\phi\right|_{H_{n}}\right)\right]=\operatorname{Ind}_{G_{n}}^{F_{n} \times K} \cdot \rho_{n}^{*}\left(\left[\bar{G}_{n} / \overline{\left(H_{n},\left.\phi\right|_{H_{n}}\right)}\right]\right) .
$$

Therefore, to prove the well-definedness of $\left[\left(F_{\infty} \times K\right) /\left(H_{\infty}, \phi\right)\right] \in \underline{\lim } A\left(F_{n}, K\right)$, we just have to show the well-definedness of

$$
\left[\bar{G}_{\infty} / \overline{\left(H_{\infty}, \phi\right)}\right] \in \lim A\left(\bar{G}_{n} \triangleright \overline{K \cap G_{n}}\right),
$$

where $\left[\bar{G}_{\infty} / \overline{\left(H_{\infty}, \phi\right)}\right]=\prod_{n}\left(y_{n}\right) \in \lim _{\longleftarrow} A\left(\bar{G}_{n} \triangleright \overline{K \cap G_{n}}\right)$ is given by

$$
y_{n}=\lim _{l \rightarrow \infty}\left(\operatorname{Res}_{\bar{G}_{n}}^{\bar{G}_{l}}\left[\bar{G}_{l} / \overline{\left(H_{l},\left.\phi\right|_{H_{l}}\right)}\right]\right) \in A\left(\bar{G}_{n} \triangleright \overline{K \cap G_{n}}\right),
$$

where the limit is taken in the $p$-adic completion $A\left(\bar{G}_{n} \triangleright \overline{K \cap G_{n}}\right)_{p}^{\wedge}$ (but, still $y_{n} \in A\left(\bar{G}_{n} \triangleright \overline{K \cap G_{n}}\right)$. The payoff of considering such a system of non-"product" relative Burnside modules is that $\overline{\left(H_{\infty}, \phi\right)}$ is a finite $p$-group; then the claim is an immediate consequence of Lemma 3.2(1) and Remark 1.3.

Proof of Proposition 3.1(2). The idea of the proof is quite similar to that of (1): Let $G$ be the subgroup of $P \times K$, generated by $(H, \psi)$ and $\operatorname{Ker} \pi_{P} \times$ $\operatorname{Ker} \pi_{K}$. Let $N=H \cap \operatorname{Ker} \pi_{P} \cap \psi^{-1}\left(\operatorname{Ker} \pi_{K}\right)$, then $N \triangleleft H$ with a finite $p$ -

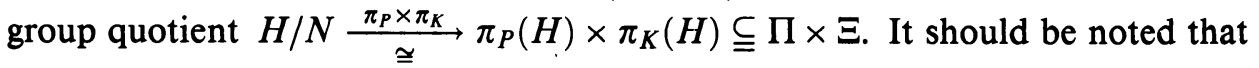


$\overline{(H, \psi)} \stackrel{\text { def }}{=}(H, \psi) /\left(N,\left.\psi\right|_{N}\right) \cong H / N$ is a finite $p$-group, rather than infinite, and is a normal subgroup of $G$ that intersects trivially with $K$. Furthermore, $\overline{(H, \psi)} \cong \overline{\left(H_{\infty}, \phi\right)}$ as $H_{\infty}$ is dense in $H$. We can also form $\bar{G}=G /\left(N,\left.\psi\right|_{N}\right)$ and we put $\rho: G \rightarrow \bar{G}$ to be the canonical quotient map. Notice also that $K \cap G=K^{\prime}$ and $(P \times K) / G \cong\left(F_{n} \times K\right) / G_{n}$ for sufficiently large $n$. Both of these follow from the facts that $H_{\infty}$ is dense in $G$ (as $H_{\infty}$ is dense in $H$ ) and that $\Pi \times \Xi \cong F_{\infty} \times K / \operatorname{Ker} \pi_{F_{\infty}} \times \operatorname{Ker} \pi_{K}$ is finite (see the proof of (1)). For such $n$, just as in (1), we have the following commutative diagram:

$$
\begin{array}{cccl}
A\left(\bar{G} \triangleright \overline{K^{\prime}}\right) \stackrel{\rho^{*}}{\longrightarrow} & A\left(G \triangleright K^{\prime}\right) \stackrel{\operatorname{Ind}_{G}^{P \times K}}{\longrightarrow} & A(P \times K \triangleright K) \\
\operatorname{Res}_{\bar{G}} \bar{G}_{n} \downarrow & \operatorname{Res}_{G_{n}}^{G} \downarrow & \operatorname{Res}_{F_{n} \times K}^{P \times K} \downarrow \\
A\left(\bar{G}_{n} \triangleright \overline{K_{n}^{\prime}}\right) \stackrel{\rho_{n}^{*}}{\longrightarrow} A\left(G_{n} \triangleright K^{\prime}\right) \stackrel{\operatorname{Ind}_{G_{n}}^{F_{n} \times K}}{\longrightarrow} & A\left(F_{n} \times K \triangleright K\right),
\end{array}
$$

which leads to maps of inverse systems:

$$
\begin{aligned}
& A\left(\bar{G} \triangleright \overline{K^{\prime}}\right) \quad \stackrel{\rho^{*}}{\longrightarrow} A\left(G \triangleright K^{\prime}\right) \quad \stackrel{\operatorname{Ind}_{G}^{P \times K}}{\longrightarrow} \quad A(P \times K \triangleright K) \\
& \lim _{n} \operatorname{Res}_{G_{n}} \bar{G}_{\square} \downarrow \quad \lim _{n} \operatorname{Res}_{G_{n}}^{G} \downarrow \quad \quad \lim _{n} \operatorname{Res}_{F_{n} \times K}^{P \times K} \downarrow \\
& \lim _{n} A\left(\bar{G}_{n} \triangleright \overline{K_{n}^{\prime}}\right) \stackrel{\lim _{n} \rho_{n}^{*}}{\longrightarrow} \lim _{\leftarrow} A\left(G_{n} \triangleright K^{\prime}\right) \stackrel{\lim _{n} \operatorname{Ind}_{G_{n}}^{F_{n} \times K}}{\longrightarrow} \lim _{\leftarrow} A\left(F_{n} \times K \triangleright K\right) .
\end{aligned}
$$

In this diagram, it is easy to see that

$$
\begin{aligned}
{[(P \times K) /(H, \psi)] } & =\operatorname{Ind}_{G}^{P \times K} \rho^{*}([\bar{G} / \overline{(H, \psi)}]), \\
{\left[\left(F_{\infty} \times K\right) /\left(H_{\infty}, \phi\right)\right] } & =\underbrace{\lim }_{n} \operatorname{Ind}_{G_{n}}^{F_{n} \times K} \underbrace{\lim \rho_{n}^{*}\left(\left[\bar{G}_{\infty} / \overline{\left(H_{\infty}, \phi\right)}\right]\right) .}_{n}
\end{aligned}
$$

Therefore, we only have to show that

$$
\left[\bar{G}_{\infty} / \overline{\left(H_{\infty}, \phi\right)}\right]=\underbrace{\lim }_{n} \operatorname{Res} \underbrace{\bar{G}}_{\bar{G}_{n}}([\bar{G} / \overline{(H, \psi)}]) .
$$

But, this is an immediate consequence of Lemma 3.2(2) and Remark 1.3.

Step (II): $A_{f}\left(F_{\infty}, K\right)$ is dense in $\lim A\left(F_{n}, K\right)_{p}^{\wedge}$. This is proved by the induction on $|\Pi|$, the order of the "connected components"of $P$ : let $\left\{\Pi^{\mu}\right\}_{\mu \in \Lambda}$ be the set of all the maximal proper subgroups of $\Pi=\pi_{F_{\infty}}\left(F_{\infty}\right)$, and let $F_{n}^{\mu}=\pi_{F_{\infty}}^{-1}\left(\Pi^{\mu}\right) \cap F_{n}$ be its inverse images. Then define $\overline{A\left(F_{n}, K\right)}$ by the exact sequence

$$
\bigoplus_{\mu \in \Lambda} A\left(F_{n}^{\mu}, K\right) \stackrel{\oplus_{\mu \in \Lambda} \operatorname{Id}_{F_{n}^{\mu}}^{F_{n}}}{\longrightarrow} A\left(F_{n}, K\right) \rightarrow \overline{A\left(F_{n}, K\right)} \rightarrow 0 .
$$

(Of course, $\overline{A\left(F_{n}, K\right)}=A\left(F_{n}, K\right)$, if $\Pi=\{e\}$.) We note that this sequence is actually that of systems as we vary $n$, and so induces

$$
\bigoplus_{\mu \in \Lambda} \stackrel{\lim }{n}_{n} A\left(F_{n}^{\mu}, K\right)_{p}^{\wedge} \stackrel{\oplus_{\mu \in \Lambda} \lim _{n} \operatorname{Ind}_{F_{n}^{\mu}}^{F_{n}}}{\longrightarrow} \underbrace{\lim }_{n} A\left(F_{n}, K\right)_{p}^{\wedge} \rightarrow \underbrace{\lim }_{n} \overline{A\left(F_{n}, K\right)_{p}^{\wedge}} \rightarrow 0 .
$$


Since

$$
(\underbrace{\lim }_{n} \operatorname{Ind}_{F_{n}^{\mu}}^{F_{n}})\left(\left[\left(F_{\infty}^{\mu} \times K\right) /\left(H_{\infty}^{\mu}, \phi\right)\right]\right)=\left[\left(F_{\infty} \times K\right) /\left(H_{\infty}^{\mu}, \phi\right)\right]
$$

it suffices to show the density of $A_{f}\left(F_{\infty}, K\right)$ in ${\underset{\lim }{\longleftarrow}}_{n} \overline{A\left(F_{n}, K\right)_{p}^{\wedge}}$ in order to proceed the inductive argument.

For this purpose, we fix a finite subgroup $F \subseteq F_{\infty}$ and we will show

$$
\operatorname{Im} A_{f}\left(F_{\infty}, K\right)_{p}^{\wedge}=\operatorname{Im} \lim _{\longleftarrow} A\left(F_{n}, K\right)_{p}^{\wedge} \subseteq \overline{A(F, K)_{p}},
$$

where $\overline{A(F, K)_{p}} \wedge$ is non-trivial only when $\pi_{F_{\infty}}(F)=\Pi$. (Recall that $\operatorname{Im} X$ stands for the image of $X$ by some given map.) Our strategy is to construct a subgroup $S$ in $\overline{A(F, K)_{p}} \wedge$ such that

$$
\operatorname{Im} \lim _{\longleftarrow} A\left(F_{n}, K\right)_{p}^{\wedge} \subseteq \operatorname{Im} S \leqq \operatorname{Im} A_{f}\left(F_{\infty}, K\right)_{p}^{\wedge}\left(\subseteq \overline{A(F, K)_{p}}\right)^{\wedge} .
$$

(Recall that $A_{f}\left(F_{\infty}, K\right)$ is the subgroup of $\lim A\left(F_{n}, K\right)$ generated by those $\left[\left(F_{\infty} \times K\right) /\left(H_{\infty}, \phi\right)\right]$ studied in $\left.(\mathrm{I}).\right)$

The following lemma is used to define $S$ :

Lemma 3.3. Let $F \subset F_{n}$ be a subgroup and let $H_{n} \subset F_{n}$ be a subgroup such that $\pi_{F_{\infty}}\left(H_{n}\right)=\Pi$, together with a group homomorphism $\phi: H_{n} \rightarrow K$. Then, in $\overline{A(F, K)}$,

$$
\operatorname{Res}_{F}^{F_{n}}\left[\left(F_{n} \times K\right) /\left(H_{n}, \phi\right)\right]=p^{l} \cdot \sum_{\left(H_{\lambda}, \phi_{\lambda}\right) \in \mathscr{L} \subset \mathscr{H}}\left[(F \times K) /\left(H_{\lambda}, \phi_{\lambda}\right)\right]
$$

for some nonnegative integer $l$, where $\phi_{\lambda}=\left.\phi\right|_{H_{\lambda}}, \mathscr{H}$ is the set of the representatives $(H, \phi)$ of the $F \times K$ conjugacy classes of this form of subgroups in $F \times K$ satisfying $\pi_{F_{\infty}}(H)=\Pi$, and $\mathscr{L}$ is a subset in $\mathscr{H}$.

Proof. As $\pi_{F_{\infty}}\left(F_{n}\right)=\pi_{F_{\infty}}\left(H_{n}\right)$, any $K$-orbit of $F_{n} \times K /\left(H_{n}, \phi\right)$ has a representative of the form $\left(t_{0} \times e\right)\left(H_{n}, \phi\right)$, where $t_{0} \in T_{n} \stackrel{\text { def. }}{\equiv} F_{n} \cap T$, and $e$ is the identity element (of $K$, in this setting). Then, when $F_{n} \times K /\left(H_{n}, \phi\right)$ is regarded as a compact $F \times K$-ENR, the isotropy subgroup $(F \times K)_{\left(t_{0} \times e\right)\left(H_{n}, \phi\right)}$ is seen to be $\left(F \cap H_{n}^{t_{0}^{-1}}, \phi \cdot c_{t_{0}}\right)(\subseteq F \times K)$, for

$$
\begin{aligned}
(f \times & k)\left(t_{0} \times e\right)\left(H_{n}, \phi\right)=\left(t_{0} \times e\right)\left(H_{n}, \phi\right) \\
& \Leftrightarrow\left(f^{t_{0}} \times k\right)\left(H_{n}, \phi\right)=\left(H_{n}, \phi\right) \\
& \Leftrightarrow\left(f^{t_{0}} \times k\right) \in\left(H_{n}, \phi\right) \\
& \Leftrightarrow f^{t_{0}} \in H_{n} \text { and } k=\phi\left(f^{t_{0}}\right) \\
& \Leftrightarrow f \in H_{n}^{t_{0}^{-1}} \text { and } k=\phi \cdot c_{t_{0}}(f) \\
& \Leftrightarrow(f \times k) \in\left(H_{n}^{t_{0}^{-1}}, \phi \cdot c_{t_{0}}\right) .
\end{aligned}
$$

We want to know when two such isotropy subgroups, which survive in $\overline{A(F, K)}$, are conjugate in $F \times K$. As $\left(F \cap H_{n}^{t_{0}^{-1}}, \phi \cdot c_{t_{0}}\right)$ survives in $\overline{A(F, K)}$ iff $\pi_{F_{\infty}}\left(F \cap H_{n}^{t_{0}^{-1}}\right)=\pi_{F_{\infty}}(F)=\Pi$, we note that two such are conjugate in $F \times K$ iff they are conjugate in $(F \cap T) \times K$. Now we are going to show the following claim in several steps: 
Sublemma 1. $(F \times K)_{\left(t_{0} \times e\right)\left(H_{n}, \phi\right)}$ and $(F \times K)_{(t \times e)\left(H_{n}, \phi\right)}$ are conjugate in $(F \cap T) \times K$ if and only if $t \in t_{0} W(F \cap T)$.

As a first step, we consider the problem: Given $t_{0} \in T_{n}$ s.t. $\pi_{F_{\infty}}\left(F \cap H_{n}^{t^{-1}}\right)=$ $\Pi$, determine all of those $t \in T_{n}$ s.t.

$$
\left((F \times K)_{\left(t_{0} \times e\right)\left(H_{n}, \phi\right)}\right)^{(f \times k)}=(F \times K)_{(t \times e)\left(H_{n}, \phi\right)},
$$

for some $f \in(F \cap T)$ and $k \in K$. Expressing these isotropy subgroups explicitly, this equality is nothing but the commutativity of the following diagram.

$$
\begin{aligned}
F & \cap H_{n}^{t_{0}^{-1}} \stackrel{c_{t_{0}^{-1}}}{\cong} F^{t_{0}} \cap H_{n} \stackrel{\phi}{\longrightarrow} K \\
\cong \downarrow c_{f} & \\
F & \cap H_{n}^{t^{-1}} \stackrel{c_{t}}{\cong} F^{t} \cap H_{n} \stackrel{\phi}{\longrightarrow} K .
\end{aligned}
$$

As $c_{t} \cdot c_{f} \cdot c_{t_{0}^{-1}}(x)=x^{t_{0}^{-1} f t}=x^{t_{0}^{-1} t f}$,

$$
\begin{aligned}
(*) \text { commutes } & \Leftrightarrow \phi\left(x^{t_{0}^{-1} t f}\right)=\phi(x)^{k} \\
& \Leftrightarrow \phi\left(x^{t_{0}^{-1} t f}\right) \phi(x)^{-1}=\phi(x)^{k} \phi(x)^{-1} \\
& \Leftrightarrow \phi\left(\left(t_{0}^{-1} t f\right)^{-1}\left(x\left(t_{0}^{-1} t f\right)\right)\right)=k^{-1}(\phi(x) k) .
\end{aligned}
$$

Of course, the problem here is that $\phi$ is defined only on $H_{n}$, not on the whole $T_{n}=F_{n} \cap T$ where $t_{0}^{-1} t f$ belongs. (Otherwise, just put $k=\phi\left(t_{0}^{-1} t f\right)$.)

Now, we wish to study the both sides of the last equality more closely:

(i) The map $x \mapsto \phi\left(\left(t_{0}^{-1} t f\right)^{-1}\left(x\left(t_{0}^{-1} t f\right)\right)\right)$

We begin by claiming that the map

$$
\begin{aligned}
F^{t_{0}} \cap H_{n} & \rightarrow H_{n} \cap T, \\
x \quad & \mapsto\left(t_{0}^{-1} t f\right)^{-1}\left({ }^{x}\left(t_{0}^{-1} t f\right)\right)
\end{aligned}
$$

factors through as

$$
\begin{aligned}
F^{t_{0}} \cap H_{n} & \rightarrow F^{t_{0}} \cap H_{n} / F^{t_{0}} \cap H_{n} \cap T \cong \Pi \rightarrow H_{n} \cap T, \\
x & \mapsto \quad x\left(F^{t_{0}} \cap H_{n} \cap T\right) \mapsto\left(t_{0}^{-1} t f\right)^{-1}\left({ }^{x}\left(t_{0}^{-1} t f\right)\right) .
\end{aligned}
$$

This is simply because both $F^{t_{0}} \cap H_{n} \cap T$ and $t_{0}^{-1} t f$ are contained in the abelian group $T_{n}$. Now, notice that this map from $\Pi$ to $H_{n} \cap T$ is a cocycle in the sense of Galois cohomology (cf. [S]). Then the obvious question here is where does $t_{0}^{-1} t f$ belong in order that the left side part of the diagram $(*)$ to be well-defined. The answer is $t_{0}^{-1} t f \in N_{T_{n}}\left(H_{n}\right)$. This is because $t_{0}^{-1} t f \in$ $C_{T_{n}}\left(H_{n}\right)$ (for $T$ is abelian), $c_{t_{0}^{-1} t f}: F^{t_{0}} \cap H_{n} \stackrel{\cong}{\longrightarrow} F^{t} \cap H_{n}$ (see $\left.(*)\right)$, and $\pi_{F_{\infty}}\left(F^{t_{0}} \cap H_{n}\right)=\pi_{F_{\infty}}\left(F^{t} \cap H_{n}\right)=\pi_{F_{\infty}}\left(H_{n}\right)$. Conversely, if $t_{o}^{-1} t f \in N_{T_{n}}\left(H_{n}\right)$, 
then we can recover the isomorphism $c_{t_{0}^{-1} t f}: F^{t_{0}} \cap H_{n} \stackrel{\cong}{\longrightarrow} F^{t} \cap H_{n}$, since $c_{t_{0}^{-1} t f}\left(H_{n}\right)=H_{n}$ and $c_{t_{0}^{-1} t f}\left(F^{t_{0}}\right)=c_{t_{0}^{-1} f t}\left(F^{t_{0}}\right)=F^{t}$. Therefore, the necessarily and sufficient condition for the left side part of the diagram $(*)$ to be welldefined is $t_{0}^{-1} t f \in N_{T_{n}}\left(H_{n}\right)$, as we claimed. Using the terminology of the Galois cohomology (cf. [S]), these data indicate that the map

$$
\begin{aligned}
\Pi \cong & F^{t_{0}} \cap H_{n} / F^{t_{0}} \cap H_{n} \cap T \rightarrow H_{n} \cap T \rightarrow \phi\left(H_{n} \cap T\right) \cong K \\
& x\left(F^{t_{0}} \cap H_{n} \cap T\right) \mapsto\left(t_{0}^{-1} t f\right)^{-1}\left({ }^{x}\left(t_{0}^{-1} t f\right)\right) \mapsto \phi\left(\left(t_{0}^{-1} t f\right)^{-1}\left({ }^{x}\left(t_{0}^{-1} t f\right)\right)\right)
\end{aligned}
$$

is a cocycle and its cohomologous class is nothing but the image of $t_{0}^{-1} t f \epsilon$ $N_{T_{n}}\left(H_{n}\right)$ under the composite

$$
N_{T_{n}}\left(H_{n}\right) \rightarrow\left(T_{n} / H_{n} \cap T_{n}\right)^{\Pi \stackrel{\delta}{\rightarrow}} H^{1}\left(\Pi, H_{n} \cap T_{n}\right) \stackrel{\phi_{*}}{\rightarrow} H^{1}\left(\Pi, \phi\left(H_{n} \cap T_{n}\right)\right),
$$

where the first map is the obvious one (recall $\pi_{F_{\infty}}\left(H_{n}\right)=\Pi$ ), $\delta$ is the connecting homomorphism, and $\phi_{*}$ is induced by $\phi$ which is $\Pi$-equivariant as $\phi$ is defined on the whole $H_{n}$ (again, recall $\pi_{F_{\infty}}\left(H_{n}\right)=\Pi$ ).

(ii) The map $x \mapsto k^{-1}(\phi(x) k)$

Notice that this map has a form of a coboundary in the sense of the nonabelian Galois cohomology (see [S]). But the obvious question here is where does $k$ belong so that the diagram $(*)$ commutes. We claim the answer is $k \in C_{K}\left(\phi\left(F \cap H_{n} \cap T\right)\right)$. Actually, this immediately follows from the fact that the left side part composite map in $(*): x \mapsto \phi\left(\left(t_{0}^{-1} t f\right)^{-1}\left(x\left(t_{0}^{-1} t f\right)\right)\right)$ factors through $\Pi \cong F^{t_{0}} \cap H_{n} / F^{t_{0}} \cap H_{n} \cap T$, as was discussed in the previous paragraph (here we used $F^{t_{0}} \cap H_{n} \cap T=F \cap H_{n} \cap T$ ).

Having studied these maps in (i) and (ii) using the Galois cohomology, we denote the kernel of the composite

$$
\begin{aligned}
N_{T_{n}}\left(H_{n}\right) \rightarrow\left(T_{n} / H_{n} \cap T_{n}\right) \stackrel{\delta}{\rightarrow} & H^{1}\left(\Pi, H_{n} \cap T_{n}\right) \\
& \downarrow \phi_{*} \\
& H^{1}\left(\Pi, \phi\left(H_{n} \cap T_{n}\right)\right) \rightarrow H^{1}\left(\Pi, \phi\left(C_{K}\left(F \cap H_{n} \cap T_{n}\right)\right)\right)
\end{aligned}
$$

by $W$. We warn the reader that $W$ might not be a group, as

$$
H^{1}\left(\Pi, C_{K}\left(\phi\left(F \cap H_{n} \cap T_{n}\right)\right)\right)
$$

is merely a set, the nonabelian Galois cohomology (notice that

$$
C_{K}\left(\phi\left(F \cap H_{n} \cap T_{n}\right)\right)
$$

is not necessarily abelian). But we do emphasize that it does not depend upon $t_{0}$ (this is actually the key to our proof). Then combining those analyses of the two composites in the diagram $(*)$ discussed in (i) and (ii), we see $(*)$ commutes iff. $t_{0}^{-1} t f \in W$. In other words,

$$
\begin{aligned}
(F \times K)_{\left(t_{0} \times e\right)\left(H_{n}, \phi\right)} & \text { and }(F \times K)_{(t \times e)\left(H_{n}, \phi\right)} \quad \text { are conjugate in }(F \cap T) \times K \\
& \Longleftrightarrow t_{0}^{-1} t f \in W \Longleftrightarrow t \in t_{0} W(F \cap T) .
\end{aligned}
$$

Of course, this is nothing but Sublemma 1. Now we are ready to prove a preliminary result toward Lemma 3.3: 
Sublemma 2. In $\overline{A(F, K)_{p}^{\wedge}}$,

$$
\operatorname{Res}_{F}^{F_{n}}\left[\left(F_{n} \times K\right) /\left(H_{n}, \phi\right)\right]=m \cdot \sum_{\left(H_{\lambda}, \phi_{\lambda}\right) \in \mathscr{L} \subset \mathscr{H}}\left[(F \times K) /\left(H_{\lambda}, \phi_{\lambda}\right)\right],
$$

where $\phi_{\lambda}=\left.\phi\right|_{H_{\lambda}}, \mathscr{H}$ is the set of the representatives $(H, \phi)$ of the $F \times K$ conjugacy classes of this form of subgroups in $F \times K$ satisfying $\pi_{F_{\infty}}(H)=\Pi$, and $\mathscr{L}$ is a subset in $\mathscr{H}$.

We begin by recalling that elements of the form $(t \times e)\left(H_{n}, \phi\right)$ form the set of representatives of $K$-orbits of such elements in $\left(F_{n} \times K\right) /\left(H_{n}, \phi\right)$ (see the beginning of this proof). Therefore, when $\left(F_{n} \times K\right) /\left(H_{n}, \phi\right)$ is regarded as a compact $F \times K$-ENR, the subset consisting of those points, whose isotropy subgroups are conjugate to $\left(F \cap H_{n}^{t_{0}^{-1}}, \phi \cdot c_{t_{0}}\right)$ such that $\pi_{F_{\infty}}\left(F \cap H_{n}^{t_{0}^{-1}}\right)=\Pi$, is given by

$$
\begin{gathered}
\left(F_{n} \times K\right) /\left(H_{n}, \phi\right)_{\left(\left(F \cap H_{n}^{t^{0}}, \phi \cdot c_{t_{0}}\right)\right)} \\
=\left(t_{0} W(F \cap T) \times K\right)\left(H_{n}, \phi\right) /\left(H_{n}, \phi\right)\left(\subseteq\left(F_{n} \times K\right) /\left(H_{n}, \phi\right)\right) \\
\quad(\because \text { Sublemma 1) } \\
=\left(\left(t_{0} W(F \cap T) H_{n}\right) \times K\right) /\left(H_{n}, \phi\right)\left(\subseteq\left(F_{n} \times K\right) /\left(H_{n}, \phi\right)\right),
\end{gathered}
$$

as an $F \times K$-space.

To apply Theorem 1.2, we notice that, for any $t \in t_{0} W(F \cap T)$,

$$
\begin{aligned}
F t H_{n} & =(F \cap T)\left(F \cap H_{n}^{t^{-1}}\right) t H_{n} \\
& =(F \cap T) t\left(F^{t} \cap H_{n}\right) H_{n}=t(F \cap T) H_{n} .
\end{aligned}
$$

Using this, we get

$$
\begin{aligned}
(F \times & K) \backslash\left[\left(F_{n} \times K\right) /\left(H_{n}, \phi\right)_{\left(\left(F \cap H_{n}^{t^{0}}, \phi \cdot c_{t_{0}}\right)\right)}\right] \\
& =(F \times K) \backslash\left(\left(t_{0} W(F \cap T) H_{n}\right) \times K\right) /\left(H_{n}, \phi\right) \\
& \cong F \backslash\left(t_{0} W(F \cap T) H_{n}\right) / H_{n} \\
& \cong\left(t_{0} W(F \cap T) H_{n}\right) /(F \cap T) H_{n} \quad(\because \text { above remark }) \\
& \stackrel{\cong}{\longrightarrow}\left(W(F \cap T) H_{n}\right) /(F \cap T) H_{n} \quad\left(\subseteq F_{n} /(F \cap T) H_{n}\right) .
\end{aligned}
$$

Notice that this is a finite set, as $F_{n}$ is a finite group. So, let us denote the order of this finite set by $m$. At this stage, it is not clear that $m$ is a $p$-power, as $W$ is not necessarily a subgroup (whereas $(F \cap T) H_{n}$ is a subgroup) of a $p$-group $F_{n}$. But $m$ is clearly independent of any particular choice of $t_{0}$, and Theorem 1.2 implies Sublemma 2.

Finally, we can complete the proof of Lemma 3.3 by showing

Sublemma 3. In Sublemma 2, $m$ is a p-power: $m=p^{l}$, for some nonnegative integer $l$.

We first remark that Sublemma 3 holds for the case of the nonrelative situation, i.e. when $K=\{e\}$. This is because, in this case, $W=N_{T^{n}}\left(H_{n}\right)$ and

$$
m=\left|N_{T^{n}}\left(H_{n}\right)(F \cap T) H_{n} /(F \cap T) H_{n}\right|,
$$


which is of $p$-power order as $N_{T^{n}}\left(H_{n}\right)(F \cap T) H_{n}$ is a subgroup of the $p$-group $F_{n}$.

The reduction of the general relative case to this nonrelative case is given by the following commutative diagram:

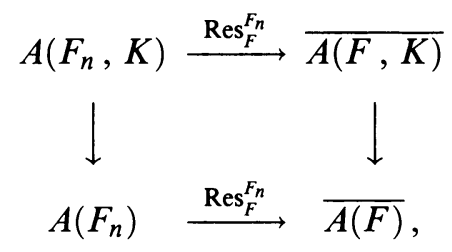

where the vertical maps are given by

$$
[X] \mapsto[X / K]=\left[X \times_{K} K\right]=\mu([X],[K])
$$

(recall that the well-definedness of $\mu$ was given in Theorem 2.3).

Suppose $\left[(F \times K) /\left(H_{\lambda}, \phi_{\lambda}\right)\right]$ shows up in $\operatorname{Res}_{F}^{F_{n}}\left[\left(F_{n} \times K\right) /\left(H_{n}, \phi\right)\right]$ with multiplicity $m$, as above. Then $\left[F / H_{\lambda}\right]$ also shows up in $\operatorname{Res}_{F}^{F_{n}}\left[F_{n} / H_{n}\right]$, with some $p$-power multiplicity (say $p^{l_{0}}$ ), as was shown above. Now, let $m^{\prime}$ be the number of those basis elements in $\overline{A(F, K)}$, which shows up in the basis expansion (in the sense of Theorem 1.2) of $\operatorname{Res}_{F}^{F_{n}}\left[\left(F_{n} \times K\right) /\left(H_{n}, \phi\right)\right]$ and is sent to $\left[F / H_{\lambda}\right]$ by the right-side vertical map. Then the commutativity of this diagram, applied to the element $\left[\left(F_{n} \times K\right) /\left(H_{n}, \phi\right)\right] \in A\left(F_{n}, K\right)$, reveals, by counting the coefficient of $\left[F / H_{\lambda}\right] \in \overline{A(F)}$ in two different ways,

$$
m \cdot m^{\prime}=p^{l_{o}} \text {. }
$$

From this, we immediately see $m$ divides $p^{l_{o}}$, and so we can write $m=p^{l}$ for some nonnegative integer $l$. This implies Sublemma 3, and thus the proof of Lemma 3.3 is finished.

\section{Definition 3.4.}

(1) Under the situation of Lemma 3.3, the element $\left[\left(F_{n} \times K\right) /\left(H_{n}, \phi\right)\right]$ is called of type $(l, \mathscr{L})$ in $F$. If $\mathscr{L}=\varnothing$, it is called of type $\varnothing$ in $F$.

(2) For each type $(l, \mathscr{L})$, define the element $e_{(l, \mathscr{L})}$ by

$$
e_{(l, \mathscr{L})}=p^{l} \cdot \sum_{(H, \phi) \in \mathscr{L} \subset \mathscr{H}}[(F \times K) /(H, \phi)] \in \overline{A(F, K)_{p}^{\wedge}} .
$$

(3) Let $\mathscr{T}$ denote the set of types $(k, \mathscr{L})$ in $F$, with nonempty $\mathscr{L} \subseteq \mathscr{H}$, s.t. $e_{(k, \mathscr{L})}$ is the image of some basis element $\left[\left(F_{n} \times K\right) /\left(H_{n}, \phi_{n}\right)\right] \in A\left(\bar{F}_{n}, K\right)$ for infinitely many $n$ 's.

(4) Define $S$ to be the $\mathbb{Z}_{p}^{\wedge}$-submodule of $\overline{A(F, K)} \wedge$, generated by those $\boldsymbol{e}_{(l, \mathscr{L})}$ such that $(l, \mathscr{L}) \in \mathscr{T}$.

(5) Let $(l, \mathscr{L})$ be an element in $\mathscr{T}$. Suppose

$$
e_{(l, \mathscr{L})}=p^{l^{\prime}} \sum_{i=1}^{j} e_{\left(l_{i}, \mathscr{L}_{i}\right)},
$$

where $e_{\left(l_{i}, \mathscr{L}_{i}\right)} \in \mathscr{T}$ for $1 \leqq i \leqq j, \quad l^{\prime}$ is a nonnegative integer (resp. positive integer), and $j \geqq 2$ (resp. $j=1$ ). Then $(l, \mathscr{L})$ is said to be decomposable. 
Otherwise, it is said to be indecomposable, and the set of indecomposable types is denoted by $\widehat{\mathscr{T}}$. Of course, $S$ is actually generated by those $e_{(l, \mathscr{L})}$ such that $(l, \mathscr{L}) \in \widehat{\mathscr{T}}$.

We are now going to prove the following main result of Step (II):

Proposition 3.5. In $\overline{A(F, K)_{p}^{\wedge}}$,

$$
\operatorname{Im} \lim A\left(F_{n}, K\right)_{p}^{\wedge} \subseteq S \subseteq\left(\operatorname{Im} A_{f}\left(F_{\infty}, K\right)\right)_{p}^{\wedge} .
$$

Consequently,

$$
\left(\operatorname{Im} A_{f}\left(F_{\infty}, K\right)\right)_{p}^{\wedge}=\operatorname{Im} \lim _{\longleftarrow} A\left(F_{n}, K\right)_{p}^{\wedge} \overline{A(F, K)_{p}^{\wedge}} .
$$

Proof of Proposition 3.5. We will show the two inclusion relations separately:

Proof of $\operatorname{Im} \lim _{\longleftarrow} A\left(F_{n}, K\right)_{p}^{\wedge} \subseteq S$.

We only have to show, for any natural number $k$,

$$
\operatorname{Im} \lim _{\longleftarrow} A\left(F_{n}, K\right)_{p}^{\wedge} \subseteq \operatorname{Im} S \quad\left(\subseteq \overline{A(F, K)} / p^{k} \overline{A(F, K)}\right),
$$

where two Im's here stand for the images in $\overline{A(F, K)} / p^{k} \overline{A(F, K)}$. Notice that, for each type $(l, \mathscr{L}) \notin \mathscr{T}$, there is some natural number $N_{0}$ such that $e_{(l, \mathscr{L})}$ is never in the image of any basis element $\left[\left(F_{n} \times K\right) /\left(H_{n}, \phi_{n}\right)\right] \in A\left(F_{n}, K\right)$ for any $n \geqq N_{0}$. Now the point is that there are only finitely many of those types $(l, \mathscr{L}) \notin \mathscr{T}$ such that $l<k$ (of course, it is the condition $l<k$, not the property $\notin \mathscr{T}$, which gives the finiteness property). Therefore, there is some natural number $N$ such that, for any $n \geqq N$, the image of any basis element $\left[\left(F_{n} \times K\right) /\left(H_{n}, \phi_{n}\right)\right] \in A\left(F_{n}, K\right)$ in $\overline{A(\bar{F}, K)} / p^{k} \overline{A(F, K)}$ is either 0 or an element of the form $e_{(l, \mathscr{L})}$ with $(l, \mathscr{L}) \in \mathscr{T}$ and $l<k$. This immediately implies the desired inclusion relationship.

Proof of $S \subseteq\left(\operatorname{Im} A_{f}\left(F_{\infty}, K\right)\right)_{p}^{\wedge}$. We fix a basis element $e_{(l, \mathscr{L})} \in S$ with $(l, \mathscr{L}) \in \widehat{\mathscr{T}}$.

We first note that there are only finitely many ways such that $e_{(l, \mathscr{L})}$ can be expressed as

$$
e_{(l, \mathscr{L})}=p^{l^{\prime}} \sum_{i=1}^{j} e_{\left(l_{i}, \mathscr{L}_{i}\right)}
$$

with $\mathscr{L}_{i} \neq \varnothing$. This is because $l_{i} \leqq l$ and $\mathscr{L}_{i} \subseteq \mathscr{L}$ for any $1 \leqq i \leqq j$.

Suppose $\left[\left(F_{n} \times K\right) /\left(H_{n}, \phi_{n}\right)\right]$ is of this fixed type $(l, \mathscr{L})$ in $F$, and suppose $F_{m}$ is a subgroup of $F_{n}$, containing $F$. Then, by Lemma 3.3, we get an expression of the form

( $) \operatorname{Res}_{F_{m}}^{F_{n}}\left[\left(F_{n} \times K\right) /\left(H_{n}, \phi_{n}\right)\right]=p^{l^{\prime}} \sum_{i=1}^{j^{\prime}}\left[\left(F_{m} \times K\right) /\left(H_{m}^{i}, \phi_{m}^{i}\right)\right] \in \overline{A\left(F_{m}, K\right)_{p}}$.

Without loss of generality, we may assume, for some $j$ with $1 \leqq j \leqq j^{\prime}$, $\left[\left(F_{m} \times K\right) /\left(H_{m}^{i}, \phi_{m}^{i}\right)\right]$ is of type $\left(l_{i}, \mathscr{L}_{i}\right)$ with $\mathscr{L}_{i} \neq \varnothing$ for $1 \leqq i \leqq j$, and, if $j<j^{\prime}$, of type $\varnothing$ for $j+1 \leqq i \leqq j^{\prime}$. Then we come up with the expression 
We now claim that there exists sufficiently large $N$ such that, for any $n \geqq$ $m \geqq N$, the only expression $(\$)$ we get by the procedure $(\nabla)$ is the trivial one: $l^{\prime}=0$ and $j=1$, i.e.

$$
e_{(l, \mathscr{L})}=e_{(l, \mathscr{L})} \text {. }
$$

Suppose this is not the case. Then there is at least one nontrivial way of expressing

$$
e_{(l, \mathscr{L})}=p^{l^{\prime}} \sum_{i=1}^{j} e_{\left(l_{i}, \mathscr{L}_{i}\right)},
$$

which is produced by $(\nabla)$ for infinitely many pairs $n \geq m$ with $m$ arbitrary large (recall that there are only finitely many ways of such an expression as (\$)). This implies that these $e_{\left(l_{i}, \mathscr{L}_{i}\right)}$ come from $A\left(F_{m}, K\right)$ for infinitely many $m$ 's, and so $\left(l_{i}, \mathscr{L}_{i}\right) \in \mathscr{T}$. Of course, this would imply that $(l, \mathscr{L})$ is decomposable, i.e. $(l, \mathscr{L}) \notin \widehat{\mathscr{T}}$, which is a contradiction.

We now set, for each $n, C_{n} \subseteq C^{f}\left(F_{n}, K\right)$ to be the set of those $\left(\left(H_{n}, \phi\right)\right) \in$ $C^{f}\left(F_{n}, K\right)$ such that $\left[\left(F_{n} \times K\right) /\left(H_{n}, \phi\right)\right]$ is of type $(l, \mathscr{L})$ in $F$. Then the above claim indicates that $\left\{C_{n}\right\}_{n \geq N}$ is an inverse system by the correspondence

$$
\begin{aligned}
C_{n} & \rightarrow C_{m} \\
\left(\left(H_{n}, \phi_{n}\right)\right) & \mapsto\left(\left(H_{m}, \phi_{m}\right)\right),
\end{aligned}
$$

where

$$
\begin{aligned}
\operatorname{Res}_{F_{m}}^{F_{n}}\left[\left(F_{n} \times K\right) /\left(H_{n}, \phi_{n}\right)\right]= & {\left[\left(F_{m} \times K\right) /\left(H_{m}, \phi_{m}\right)\right] } \\
& \left(+\sum_{i=2}^{j^{\prime}}\left[\left(F_{m} \times K\right) /\left(H_{m}^{i}, \phi_{m}^{i}\right)\right]\right) \in \overline{A\left(F_{m}, K\right)_{p}^{\wedge}} .
\end{aligned}
$$

Here $\left(H_{m}, \phi_{m}\right)$ is of the type $(l, \mathscr{L})$ in $F$, and in the second summation, which might not show up, every term $\left(H_{m}^{i}, \phi_{m}^{i}\right)\left(2 \leqq i \leqq j^{\prime}\right)$ is of type $\varnothing$. In particular, this shows each $C_{n}(n \geqq N)$ is a nonempty (finite) set. Thus, as $\left\{C_{n}\right\}_{n \geq N}$ is seen to be an inverse system of nonempty finite sets,

$$
\underset{n \geq N}{\lim _{n}} C_{n} \neq \varnothing \text {. }
$$

Therefore, after applying appropriate conjugations, we can find a system of type $(l, \mathscr{L})$ subgroups $\left\{\left(H_{n}, \phi_{n}\right)\right\}_{n \geq N}$ such that

$$
\begin{aligned}
\left(H_{n}, \phi_{n}\right) & \subset F_{n} \times K, \\
H_{n+1} \cap F_{n} & =H_{n}, \\
\left.\phi_{n+1}\right|_{H_{n}} & =\phi_{n}
\end{aligned}
$$

for all $n \geq N$. Now, just set

$$
H_{\infty}=\bigcup_{n} H_{n}, \quad \phi_{\infty}=\bigcup_{n} \phi_{n}\left(\text { so }\left.\phi_{\infty}\right|_{H_{n}}=\phi_{n}\right) .
$$

Then, from Proposition 3.1(1) and Lemma 3.2(1), we get immediately

$$
\left(\stackrel{\lim }{\longrightarrow} \operatorname{Res}_{F}^{F_{n}}\right)\left(\left[\left(F_{\infty} \times K\right) /\left(H_{\infty}, \phi_{\infty}\right)\right]=e_{(l, \mathscr{L})} \in \overline{A\left(F_{m}, K\right)} .\right.
$$


Since $e_{(l, \mathscr{L})}$ is a basis element of $S$, this completes the proof.

Step (III): Representation Theory. Subgroups of $F_{\infty}$ are studied by the integral representation theory, through the Pontryagin duality. This was first observed by Feshbach [F2]. We now recall the elementary property of the Pontryagin duality.

(1) For a locally compact Lie group $G$, the Pontryagin dual $G^{*}$ is defined by

$$
G^{*}=\operatorname{Hom}_{\text {Continuous }}\left(G, S^{1}\right) \text {. }
$$

(2) $G^{* *}=G$.

(3) For each closed subgroup $H \subset G$, define $H_{*}$ as a subgroup of $G^{*}$ consisting of those $\phi: G \rightarrow S^{1}$ with $\phi(H)=1$.

(4) $H_{*} \cong(G / H)^{*}$ and $H_{* *}=H$.

(5) $T^{*}$, the Pontryagin dual of the torus $T$ with $\Pi$-action, is a finitely generated $\mathbb{Z} \Pi$-module.

(6) Let $T_{\infty}^{*}$ be the subgroup consisting of elements of $p$-power order in the torus $T$ with $\Pi$-action, as usual. Then $T_{\infty}^{*}$, the Pontryagin dual of $T_{\infty}$ with $\Pi$-action, is the $p$-completion of $T^{*}$.

Now the representation theory we need is the following:

Lemma 3.6. (1) Let $H_{*}$ be a finitely generated $\mathbb{Z}_{p} \Pi$-submodule of $T_{\infty}^{*}$, such that $H_{*} \otimes \mathbb{Q}_{p}$ is isomorphic to the p-completion of a $\mathbb{Q} \Pi$-module. Then for every $n$ there is an automorphism $\alpha_{n}$ of $T_{\infty}^{*}$, inducing the identity on $T_{\infty}^{*} / p^{n} \cdot T_{\infty}^{*}$, such that $\alpha_{n}\left(H_{n}\right)$ is the p-completion of a $\mathbb{Z} \Pi$-submodule $K_{*}^{n}$ of $T^{*}$.

(2) Let $F$ be a finite group and let $S$ and $T$ be finitely generated $\mathbb{Z} F$-modules which are free over $\mathbb{Z}$. Then

$$
\operatorname{Hom}_{\mathbb{Z} F}(S, T) \rightarrow \operatorname{Hom}_{\mathbb{Z}_{p}^{\wedge} F}\left(S_{p}^{\wedge}, T_{p}^{\wedge}\right)
$$

has a dense image.

Proof. (1) was essentially used in [F2], but was singled out explicitly in [B]. (2) is proved in 2-e of $[R]$. Of course, the general reference of these results is [CR].

This is used in the following:

Proposition 3.7. Suppose that all the irreducible $\mathbb{Q} \Pi^{\prime}$-summands in the $\Pi^{\prime}$-action on $T^{*} \otimes \mathbb{Q}$ remain irreducible after the p-adic completion for any subgroup $\Pi^{\prime} \subset \Pi$. Then for any $\left[\left(F_{\infty} \times K\right) /\left(H_{\infty}, \phi_{\infty}\right)\right] \in A_{f}\left(F_{\infty} \times K\right)$ and $n \in \mathbb{N}$ such that $\pi_{P}\left(H_{n}\right)=\pi_{P}\left(H_{\infty}\right) \subseteq \Pi$, there is a closed subgroup $H \subset P$ and a continuous homomorphism $\psi: H \rightarrow K$ such that

$$
\begin{aligned}
(\underbrace{\lim }_{m} \operatorname{Res}_{F_{n}}^{F_{m}})\left(\left[\left(F_{\infty} \times K\right) /\left(H_{\infty}, \phi_{\infty}\right)\right]\right) & =\operatorname{Res}_{F_{n}}^{P}([(P \times K) /(H, \psi)]) \\
& \in A\left(F_{n}, K\right) .
\end{aligned}
$$

Proof. Let $P^{\prime}=P \cap \pi_{P}^{-1}\left(\pi_{P}\left(H_{\infty}\right)\right)$ and $F_{n}^{\prime}=F_{n} \cap \pi_{P}^{-1}\left(\pi_{P}\left(H_{\infty}\right)\right)$ for any $n$. 
Then we have the following commutative diagram:

$$
\begin{aligned}
& A\left(P^{\prime}, K\right) \stackrel{\lim _{m} \operatorname{Res}_{F_{m}^{\prime}}^{P^{\prime}}}{\longrightarrow} \lim _{m} A\left(F_{m}^{\prime}, K\right) \stackrel{\lim _{m} \operatorname{Res}_{F_{n}^{\prime}}^{F_{m}^{\prime}}}{\longrightarrow} A\left(F_{n}^{\prime}, K\right) \\
& \operatorname{Ind}_{P^{\prime}}^{p} \downarrow \quad \lim _{m} \operatorname{Idd}_{F_{m}^{\prime}}^{F_{m}} \downarrow \quad \operatorname{Ind}_{F_{n}^{\prime}}^{F_{n}} \downarrow \\
& A(P, K) \stackrel{\lim _{m} \operatorname{Res}_{F_{m}}^{P}}{\longrightarrow} \lim _{-m} A\left(F_{m}, K\right) \stackrel{\lim _{m} \operatorname{Res}_{F_{n}}^{F_{m}}}{\longrightarrow} A\left(F_{n}, K\right) .
\end{aligned}
$$

Notice that

$$
\left({\underset{m}{\lim }}_{\operatorname{Ind}_{F_{m}^{\prime}}^{F_{m}}}\right)\left(\left[\left(F_{\infty}^{\prime} \times K\right) /\left(H_{\infty}, \phi_{\infty}\right)\right]\right)=\left[\left(F_{\infty} \times K\right) /\left(H_{\infty}, \phi_{\infty}\right)\right] .
$$

Therefore, the commutativity of the diagram implies that it suffices to find a closed subgroup $H \subseteq P^{\prime}$ and a continuous homomorphism $\psi: H \rightarrow K$ s.t.

$$
\begin{aligned}
\left(\stackrel{\lim }{m}_{m} \operatorname{Res}_{F_{n}^{\prime}}^{F_{m}^{\prime}}\right)\left(\left[\left(F_{\infty}^{\prime} \times K\right) /\left(H_{\infty}, \phi_{\infty}\right)\right]\right) & =\operatorname{Res}_{F_{n}^{\prime}}^{P^{\prime}}\left(\left(P^{\prime} \times K\right) /(H, \psi)\right) \\
& \in A\left(F_{n}^{\prime}, K\right) .
\end{aligned}
$$

For this, we apply two claims of Lemma 3.6 in two steps: (i) Find an automorphism $\alpha$ of $F_{\infty}^{\prime}=\bigcup_{n \in \mathbb{N}} F_{n}^{\prime}$ fixing $F_{n}^{\prime}$ such that $\alpha\left(H_{\infty}\right)=H \cap F_{\infty}^{\prime}$ for for some closed subgroup $H \subset P^{\prime}$; (ii) Find a continuous homomorphism $\psi: H \rightarrow K$ such that $\left.\psi\right|_{\alpha\left(H_{m}\right)}=\left.\phi_{\infty} \cdot \alpha^{-1}\right|_{\alpha\left(H_{m}\right)}$ for some sufficiently large $m$ so that

$$
\begin{gathered}
\left(\stackrel{\lim }{m} \operatorname{Res}_{F_{n}^{\prime}}^{F_{m}^{\prime}}\right)\left(\left[\left(F_{\infty}^{\prime} \times K\right) /\left(\alpha\left(H_{\infty}\right), \phi_{\infty} \cdot \alpha^{-1}\right)\right]\right) \\
=\operatorname{Res}_{F_{n}^{\prime}}^{P^{\prime}}\left(\left(P^{\prime} \times K\right) /(H, \psi)\right) \in A\left(F_{n}^{\prime}, K\right) .
\end{gathered}
$$

These would prove the claim by Proposition 3.1(2) and the following commutative diagram:

$$
\begin{aligned}
& A\left(P^{\prime}, K\right) \stackrel{\lim _{m} \operatorname{Res}_{F_{m}^{\prime}}^{P^{\prime}}}{\longrightarrow} \varliminf_{m} A\left(F_{m}^{\prime}, K\right) \stackrel{\alpha^{*}}{\longrightarrow} \varliminf_{m} A\left(F_{m}^{\prime}, K\right) \\
& \| \quad \lim _{m} \operatorname{Res}_{F_{n}^{\prime}}^{F_{m}^{\prime}} \downarrow \quad \lim _{m} \operatorname{Res}_{F_{n}^{\prime}}^{F_{m}^{\prime}} \downarrow \\
& A\left(P^{\prime} . K\right) \stackrel{\operatorname{Res}_{F_{n}^{\prime}}^{P^{\prime}}}{\longrightarrow} \quad A\left(F_{n}^{\prime}, K\right)=A\left(F_{n}^{\prime}, K\right) \text {, }
\end{aligned}
$$

where

$$
\alpha_{*}\left(\left[\left(F_{\infty}^{\prime} \times K\right) /\left(\alpha\left(H_{\infty}\right), \phi_{\infty} \cdot \alpha^{-1}\right)\right]\right)=\left(\left[\left(F_{\infty}^{\prime} \times K\right) /\left(H_{\infty}, \phi_{\infty}\right)\right]\right) .
$$

For (i), it suffices to find a $\Pi^{\prime} \stackrel{\text { def. }}{=} \pi_{P}\left(H_{\infty}\right)(\subseteq \Pi)$-invariant closed subgroup $A$ such that

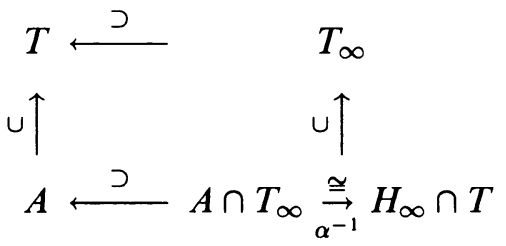


commutes for some automorphism $\alpha$ of $F_{\infty}$, fixing $F_{n}$. This is because $H$ is taken to be the closed subgroup of $P^{\prime}$, generated by $A$ and $\alpha\left(H_{n}\right)$. Now, through the Pontryagin duality, this is equivalent to the commutativity of the following diagram:

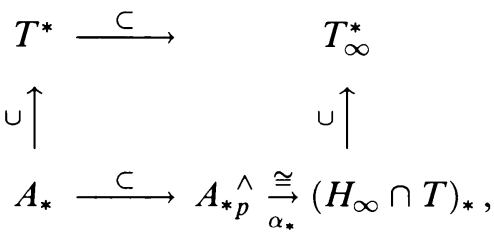

where $\alpha_{*}$ is a $\mathbb{Z}_{p}^{\wedge} \Pi^{\prime}$-automorphism of $T_{\infty}^{*}$, inducing an identity on $T_{\infty}^{*} / p^{n}$. $T_{\infty}^{*}$, To find such a submodule $A_{*}$ and an automorphism $\alpha_{*}$, it suffices to show $\left(H_{\infty} \cap T\right)_{*} \otimes \mathbb{Q}$ is the $p$-completion of a $\mathbb{Q} \Pi^{\prime}$-module, thanks to Lemma 3.6(1). In this situation we can write $T \otimes \mathbb{Q}=\bigoplus_{i} V_{i}$ for some irreducible $\mathbb{Q} \Pi^{\prime}$-modules $V_{i}$ 's. But each $V_{i p}^{\wedge}$ is still an irreducible $\mathbb{Q}_{p} \Pi^{\prime}$-module by the assumption. Therefore $\left(H_{\infty} \cap T\right)_{*} \otimes \mathbb{Q}$, which is a $\mathbb{Q} \Pi^{\prime}$-submodule of $T_{\infty}^{*} \otimes \mathbb{Q}=$ $\bigoplus_{i} V_{i p}^{\wedge}$, is isomorphic to a finite combination of the irreducible summands $V_{i p}^{\wedge}$ 's. As each $V_{i p}^{\wedge}$ is the $p$-completion of $V_{i}$, this immediately implies that $\left(H_{\infty} \cap T\right)_{*} \otimes \mathbb{Q}$ is the $p$-completion of a $\mathbb{Q} \Pi^{\prime}$-submodule, as desired.

For (ii), we first notice that the above $H$ and $\alpha\left(H_{\infty}\right)$ are a $p$-toral and its dense locally $p$-subgroup. Therefore, we may apply the following Lemma 3.8 to obtain the isomorphism $A\left(F_{m}^{\prime}, \alpha\left(H_{\infty}\right)\right) \stackrel{\cong}{\rightrightarrows} A\left(F_{m}^{\prime}, H\right)$ for any $m$. These isomorphisms are combined to give an isomorphism $\lim _{m} A\left(F_{m}^{\prime}, \alpha\left(H_{\infty}\right)\right) \stackrel{\rightrightarrows}{\rightrightarrows}$ $\stackrel{\lim }{\longleftarrow}_{m} A\left(F_{m}^{\prime}, H\right)$, so we can talk about the element

$$
\left[F_{\infty}^{\prime} \times \alpha\left(H_{\infty}\right) /\left(\alpha\left(H_{\infty}\right), \text { identity }\right)\right] \in \underbrace{\lim }_{m} A\left(F_{m}^{\prime}, \alpha\left(H_{\infty}\right)\right)
$$

(see Proposition 3.1(1)) and the isomorphism

$$
\begin{gathered}
\left(\phi_{\infty} \cdot \alpha^{-1}\right)_{*}\left(\left[F_{\infty}^{\prime} \times \alpha\left(H_{\infty}\right) /\left(\alpha\left(H_{\infty}\right), \text { identity }\right)\right]\right) \\
=\left[\left(F_{\infty}^{\prime} \times K\right) /\left(\alpha\left(H_{\infty}\right), \phi_{\infty} \cdot \alpha^{-1}\right)\right] .
\end{gathered}
$$

For this important element, we also get a finite expansion

$$
\begin{aligned}
& (\underbrace{\lim }_{m} \operatorname{Res}_{F_{n}^{\prime}}^{F_{m}^{\prime}})\left(\left[F_{\infty}^{\prime} \times \alpha\left(H_{\infty}\right) /\left(\alpha\left(H_{\infty}\right), \text { identity }\right)\right]\right) \\
& \quad=\sum_{i} n_{i}\left[F_{n}^{\prime} \times \alpha\left(H_{\infty}\right) /\left(F_{n}^{\prime} \cap \alpha\left(H_{\infty}\right)^{f_{i}}, c_{f_{i}^{-1}}\right)\right],
\end{aligned}
$$

where $n_{i} \in \mathbb{N}$ and $f_{i} \in F_{\infty}^{\prime}$. Then, these two equalities and the commutativity of the diagram

$$
\begin{aligned}
& \varliminf_{m} A\left(F_{m}^{\prime}, \alpha\left(H_{\infty}\right)\right) \stackrel{\phi_{\infty}^{*} \alpha^{-1}}{\longrightarrow} \lim _{m} A\left(F_{m}^{\prime}, K\right) \\
& \lim _{m} \operatorname{Res}_{F_{n}^{\prime}}^{F_{m}^{\prime}} \downarrow \quad \quad \quad \lim _{m} \operatorname{Res}_{F_{n}^{\prime}}^{F_{m}^{\prime}} \downarrow \\
& A\left(F_{n}^{\prime}, \alpha\left(H_{\infty}\right)\right) \stackrel{\phi_{\infty} \cdot \alpha^{-1}}{\longrightarrow} A\left(F_{n}^{\prime}, K\right)
\end{aligned}
$$


immediately imply

$$
\begin{aligned}
& (\underbrace{\lim }_{m} \operatorname{Res}_{F_{n}^{\prime}}^{F_{m}^{\prime}})\left(\left[F_{\infty}^{\prime} \times K /\left(\alpha\left(H_{\infty}\right), \phi_{\infty} \cdot \alpha^{-1}\right)\right]\right) \\
& \quad=\sum_{i} n_{i}\left[F_{n}^{\prime} \times K /\left(F_{n}^{\prime} \cap \alpha\left(H_{\infty}\right)^{f_{i}}, \phi_{\infty} \cdot \alpha^{-1} \cdot c_{f_{i}^{-1}}\right)\right] \in A\left(F^{\prime} n, K\right) .
\end{aligned}
$$

Here $\phi_{\infty} \cdot \alpha^{-1} \cdot c_{f_{i}^{-1}}$ factors through as

$$
F_{n}^{\prime} \cap \alpha\left(H_{\infty}\right)^{f_{i}} \stackrel{c_{f_{i}^{-1}}}{\longrightarrow} F_{n}^{\prime f_{i}^{-1}} \cap \alpha\left(H_{\infty}\right) \stackrel{\phi_{\infty} \cdot \alpha^{-1}}{\longrightarrow} K .
$$

But, as there are only finitely many these $f_{i}$ 's of $F_{\infty}^{\prime}$, there is some sufficiently large $m \in \mathbb{N}$ s.t. $F_{n}^{\prime f_{i}^{-1}} \subseteq F_{m}^{\prime}$ for all $i$. Thus,

$$
{F_{n}^{\prime}}^{f_{i}^{-1}} \cap \alpha\left(H_{\infty}\right) \subseteq F_{m}^{\prime} \cap \alpha\left(H_{\infty}\right)=\alpha\left(F_{m}^{\prime}\right) \cap \alpha\left(H_{\infty}\right)=\alpha\left(H_{m}\right)
$$

for all these $f_{i}$ 's. Therefore, these indicate the following: As far as $\phi_{\infty} \cdot \alpha^{-1}$ concerns,

$$
\left(\stackrel{\lim }{m}_{m} \operatorname{Res}_{F_{n}^{\prime}}^{F_{m}^{\prime}}\right)\left(\left[F_{\infty}^{\prime} \times K /\left(\alpha\left(H_{\infty}\right), \phi_{\infty} \cdot \alpha^{-1}\right)\right]\right) \in A\left(F_{n}^{\prime}, K\right)
$$

depends only upon $\left.\phi_{\infty} \cdot \alpha^{-1}\right|_{\alpha\left(H_{m}\right)}$.

Now, by Lemma 3.6 (2) and the Pontryagin duality argument as in (i), we can find a continuous homomorphism $\psi: H \rightarrow K$ such that $\left.\psi\right|_{\alpha\left(H_{m}\right)}=\phi_{\infty}$. $\left.\alpha^{-1}\right|_{\alpha\left(H_{m}\right)}$. Of course, the above argument implies

$$
\begin{aligned}
& (\underbrace{\lim }_{m} \operatorname{Res}_{F_{n}^{\prime}}^{F_{m}^{\prime}})\left(\left[F_{\infty}^{\prime} \times K /\left(\alpha\left(H_{\infty}\right), \phi_{\infty} \cdot \alpha^{-1}\right)\right]\right) \\
& \quad=(\underbrace{\lim }_{m} \operatorname{Res}_{F_{n}^{\prime}}^{F_{m}^{\prime}})\left(\left[F_{\infty}^{\prime} \times K /\left(\alpha\left(H_{\infty}\right),\left.\psi\right|_{\alpha\left(H_{\infty}\right)}\right)\right]\right) \in A\left(F_{n}^{\prime}, K\right) .
\end{aligned}
$$

On the other hand, Proposition 3.1(2) implies

$$
\left[F_{\infty}^{\prime} \times K /\left(\alpha\left(H_{\infty}\right),\left.\psi\right|_{\alpha\left(H_{\infty}\right)}\right)\right]=\underset{n}{\lim } \operatorname{Res}_{F_{n}^{\prime}}^{P^{\prime}}\left(\left[\left(P^{\prime} \times K\right) /(H, \psi)\right]\right) .
$$

Now the claim (ii) follows immediately, and thus we have finished the proof of Proposition 3.7.

In the course of the above proof, we used the following:

Lemma 3.8. Let $F$ be a finite p-group and let $Q$ be a p-toral which is built by the extension

$$
1 \rightarrow T \rightarrow Q \rightarrow \Omega \rightarrow 1
$$


where $T$ is a torus and the connected component of $Q$ and $\Omega$ is a p-group. Suppose $Q_{\infty} \stackrel{\text { def. }}{=} \bigcup_{n} Q_{n}$ is a dense locally p-subgroup of $Q$ such that

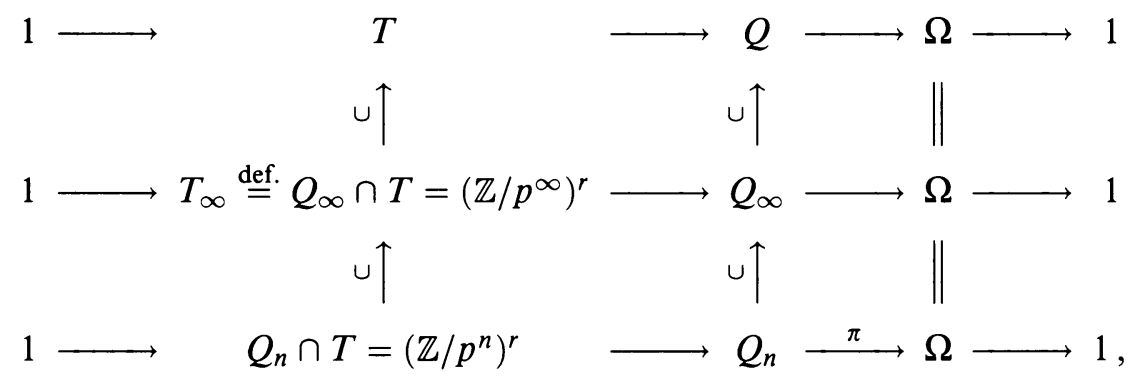

where $r$ is the rank of $T$, and so the left-upper map $\left(\mathbb{Z} / p^{\infty}\right)^{r} \rightarrow T$ is the inclusion of the subset consisting of all p-power order elements.

Then the canonical map

$$
A\left(F, Q_{\infty}\right) \stackrel{\text { def. }}{=} \underset{n}{\lim } A\left(F, Q_{n}\right) \rightarrow A(F, Q)
$$

is an isomorphism.

Proof. We begin by showing the surjectivity of this map. Notice that any basis element of $A(F, Q)$ is of the form $[F \times Q /(H, \phi)]$. As $\phi(H)$ is a finite $p$ group of $Q$, we can find another dense locally $p$-subgroup $Q_{\infty}^{\prime}$ such that $\phi(H) \subset Q_{\infty}^{\prime}$. But $Q_{\infty}^{\prime}$ is conjugate to $Q_{\infty}$ in $Q$ [F2]. So the surjectivity follows.

To prove the injectivity, it suffices to show any two $p$-subgroups of $Q_{\infty}$, which are conjugate in $Q$ (then automatically they are conjugate in $T$ ), are conjugate in $Q_{\infty}$. For this, let us suppose $S$ is a $p$-group such that both $S$ and $S^{t}$ are subgroups of $Q_{\infty}$, where $t \in T$. Then, as $S^{t} \subset Q_{\infty}$,

$$
t Q_{\infty} \in\left(Q / Q_{\infty}\right)^{S} \text {. }
$$

Also, as $S \subset Q_{\infty}$, the Key Lemma implies

$$
\left(Q / Q_{\infty}\right)^{S}=\left(T / T_{\infty}\right)^{S},
$$

where $\left(Q / Q_{\infty}\right)^{S}$ is taken with respect to the standard left $S$-multiplication on $Q / Q_{\infty}$, and $\left(T / T_{\infty}\right)^{S}$ is taken with respect to the conjugate $S$-action on $T / T_{\infty}$. The reason we prefer $\left(T / T_{\infty}\right)^{S}$ to $\left(Q / Q_{\infty}\right)^{S}$ is the accessibility of the connecting homomorphism

$$
\delta:\left(T / T_{\infty}\right)^{S} \rightarrow H^{1}\left(S, T_{\infty}\right),
$$

which we claim to be trivial. This is follows from the following: (i) $\left(T / T_{\infty}\right)^{S}$ is a $\mathbb{Z}\left[\frac{1}{p}\right]$-module; (ii) $H^{1}\left(S, T_{\infty}\right)$ is $|S|$-torsion. So the composite

$$
t Q_{\infty} \in\left(Q / Q_{\infty}\right)^{S}=\left(T / T_{\infty}\right)^{S} \stackrel{\delta}{\longrightarrow} H^{1}\left(S, T_{\infty}\right)
$$

is trivial. Therefore, the cocycle

$$
\delta\left(t Q_{\infty}\right): s \mapsto t^{-1}\left({ }^{s} t\right), \quad s \in S,
$$

is expressed as a principal crossed homomorphism

$$
s \mapsto u^{-1}\left({ }^{s} u\right), \quad s \in S,
$$


for some $u \in T_{\infty}$. In particular, this implies $S^{t}=S^{u}$. Thus, $S$ and $S^{t}$ are conjugate by an element $u \in T_{\infty} \subset Q_{\infty}$, as was desired.

Now we are finally ready to prove our main theorem:

Theorem 3.9. Let $L$ and $K$ be compact Lie groups, then

$$
\alpha_{p}^{\wedge}: A(L, K)_{p}^{\wedge} \rightarrow\left\{B L_{+}, B K_{+}\right\}_{p}^{\wedge},
$$

is dense with respect to the skeletal filtration of the target, if $L$ satisfies the assumption of the Feshbach density theorem, i.e. if either one of the following two conditions holds:

(1) $p$ is odd.

(2) $p=2$ and $\rho: \Pi \rightarrow G l(n, \mathbb{Z})$, given by the action of a 2-Sylow subgroup $\Pi$ of the Weyl group on the maximal torus, does not originate at a generalized quaternion group of order $2^{n}, n \geq 4$. Or if all the irreducible $\mathbb{Q} \Pi^{\prime}$-summands in the $\Pi^{\prime}$-action on $T^{*} \otimes \mathbb{Q}$ remain irreducible after the 2-adic completion for any 2-subgroup $\Pi^{\prime} \subset W$. In particular, this condition holds if $n<8$ or if no subquotient of $W$ is isomorphic to the quaternion group of order 16 . Here $\Pi$ is the 2-Sylow subgroup of the Weyl group of $L$ and $T$ is a maximal torus of $L$. Proof. As was noted in the introduction, the problem is reduced to the density problem of the map

$$
\underset{n}{\lim } \operatorname{Res}_{F_{n}}^{P}: A(P, K)_{p}^{\wedge} \rightarrow \underset{n}{\lim } A\left(F_{n}, K\right)_{p}^{\wedge} .
$$

But, this is an immediate consequence of Step (I), Step (II), and Step (III) (Proposition 3.7). (See the beginning of $\$ 3$.)

Corollary 3.10. If $L$ is a central extension of a finite group by a torus:

$$
0 \rightarrow T \rightarrow L \rightarrow G \rightarrow 1,
$$

then the homomorphism

$$
\alpha_{p}^{\wedge}: A(L, K)_{I(L)}^{\wedge} \rightarrow\left\{B L_{+}, B K_{+}\right\}
$$

is injective and has a dense image.

Proof. As usual, we may reduce to the local situation. Then the density part follows from Theorem 3.9. To prove the injectivity, let $P \subset L$ be a finite index subgroup corresponding to a $p$-Sylow subgroup of $G$. We only have to show the injectivity of

$$
\underbrace{\lim }_{n} \operatorname{Res}_{F_{n}}^{P}: A(P, K)_{p}^{\wedge} \rightarrow \underset{n}{\lim } A\left(F_{n}, K\right)_{p}^{\wedge} .
$$

Now the point is $U \supset T$ if $(U, \psi) \in C^{f}(P, K)$. This is because the $G$ action on $T$ is trivial. From this, we immediately see

$$
\operatorname{Res}_{F_{n}}^{P}([(P \times K) /(U, \psi)])=\left[\left(F_{n} \times K\right) /\left(U \cap F_{n},\left.\psi\right|_{U \cap F_{n}}\right)\right] .
$$

(Of course, $n$ is sufficiently large so that $F_{n}$ surjects onto a $p$-Sylow subgroup of $G$.) Now the injectivity follows quite easily.

Corollary 3.11.

$$
A(L)_{I(L)}^{\wedge} \rightarrow \pi_{S}^{0}\left(B L_{+}\right) \quad \text { is an isomorphism }
$$


$\Longleftrightarrow L$ is a central extension of a finite group $G$ by a torus:

$$
0 \rightarrow T \rightarrow L \rightarrow G \rightarrow 1 \text {. }
$$

Proof. $\Longrightarrow$ was shown in [M1]. $\Longleftarrow$ follows from Corollary 3.10 and the fact that $A(L)$ is finitely generated.

Remark 3.12. This result was announced in the introduction of [M1].

\section{APPENDIX}

In this appendix, we will prove a couple of (not surprising) claims stated (and used) in the introduction.

Let $P$ and $K$ be maximal $p$-tori of $L$ and $H$, respectively, and let $I(P, K)$ be the kernel of $\operatorname{Res}_{\{e\}}^{P}: A(P, K) \rightarrow A(\{e\}, K) \cong \mathbb{Z}$. Furthermore, let $F_{i}$ 's be increasing finite $p$-subgroups of $P$, such that $\bigcup_{i} F_{i} \subset P$ is dense (see Reduction in $\S 0$ and [F2], [M1], [N]). Then, consider the following various density properties:

(1) Density of $A(L, H) \rightarrow \varliminf_{n}\left\{B L_{+}^{(n)}, B H_{+}\right\}_{\text {finite }}^{\wedge}$,

$\left(2_{p}\right)$ Density of $A(L, H) \rightarrow \lim _{n}\left\{B L_{+}^{(n)}, B H_{+}\right\}_{p}^{\wedge}$,

$\left(3_{p}\right)$ Density of $A(P, K) \rightarrow \lim _{n}\left\{B P_{+}^{(n)}, B K_{+}\right\}_{p}^{\wedge}$,

$\left(4_{p}\right)$ Density of $I(P, K) \rightarrow \lim _{n}\left\{B P^{(n)}, B K_{+}\right\}_{p}^{\wedge}$,

$\left(5_{p}\right)$ Density of $I(P, K) \rightarrow \varlimsup_{\varlimsup_{i}}^{n}\left\{B F_{i}, B K_{+}\right\}_{p}^{\wedge}$,

$\left(6_{p}\right)$ Density of $A(P, K) \rightarrow \varlimsup_{\lim _{i}}\left\{B F_{i_{+}}, B K_{+}\right\}_{p}^{\wedge}$,

( $\left.7_{p}\right)$ Density of $A(P, K) \rightarrow \varliminf_{i}^{\lim } A\left(B F_{i}, B K\right)_{p}^{\wedge}$.

Here all the maps are canonical ones induced by $\alpha, X^{(n)}$ stands for the $n$ skeleton of $X$, and the topology of the target is the inverse limit topology of the profinite topology (resp. $p$-adic topology) on the relevant $\{-,-\}$ groups in (1) (resp. $\left.(2 p)-\left(7_{p}\right)\right)$. Now, we are going to show

Local-Global. $\left(2_{p}\right)$ for any $p \Longrightarrow(1)$.

Reduction. $\left(7_{p}\right) \Longrightarrow\left(2_{p}\right)$.

Of course, Theorem 3.9 claims $\left(7_{p}\right)$ for any odd prime $p$.

Proof of Local-Global. This is standard. Given a natural number $N$, write $N=\prod_{p \mid N} p^{\nu_{p}(N)}$. Then consider the commutative diagram

$$
\begin{gathered}
A(L, H) \longrightarrow \lim _{n}\left\{B L_{+}^{(n)}, B H_{+}\right\}_{\text {finite }}^{\wedge} \longrightarrow \frac{\left\{B L_{+}^{(n)}, B H_{+}\right\}}{N\left\{B L_{+}^{(n)}, B H_{+}\right\}} \\
\downarrow \\
A(L, H) \longrightarrow \lim _{n}\left\{B L_{+}^{(n)}, B H_{+}\right\}_{p}^{\wedge} \longrightarrow \frac{\left\{B L_{+}^{(n)}, B H_{+}\right\}}{p^{\nu_{p}(N)}\left\{B L_{+}^{(n)}, B H_{+}\right\}},
\end{gathered}
$$

where $\pi_{p}$ is the canonical projection, and the middle vertical map exists because $\left\{B L_{+}^{(n)}, B H_{+}\right\}$is finitely generated. Let $x \in\left\{B L_{+}^{(n)}, B H_{+}\right\} / N\left\{B L_{+}^{(n)}, B H_{+}\right\}$ be an element which comes from $\lim _{n}\left\{B L_{+}^{(n)}, B H_{+}\right\}_{\text {finite }}^{\wedge}$, and let $x_{p}$ be 
its image in $\left\{B L_{+}^{(n)}, B H_{+}\right\} / p^{\nu_{p}(N)}\left\{B L_{+}^{(n)}, B H_{+}\right\}$. Then, by $\left(2_{p}\right)$, there is some $a_{p} \in A(L, H)$ which goes to $x_{p}$. To slightly modify $a_{p}$, choose some integer $N_{p}$ s.t. $N_{p} \equiv 1\left(\bmod p^{\nu_{p}(N)}\right), N_{p} \equiv 0\left(\bmod \prod_{q \neq p} q^{\nu_{q}(N)}\right)$. Then set $a_{p}^{\prime}=N_{p} a_{p}$. Notice that $a_{p}^{\prime}$ also goes to $x_{p}$ just like $a_{p}$, but its real advantage is that it goes to 0 under the composite

$$
\begin{aligned}
A(L, H) & \rightarrow\left\{B L_{+}^{(n)}, B H_{+}\right\} / N\left\{B L_{+}^{(n)}, B H_{+}\right\} \\
& \stackrel{\pi_{q}}{\rightarrow}\left\{B L_{+}^{(n)}, B H_{+}\right\} / q^{\nu_{q}(N)}\left\{B L_{+}^{(n)}, B H_{+}\right\}
\end{aligned}
$$

for any $q \neq p$. Finally, set $a=\sum_{p \mid N} a_{p}^{\prime} \in A(L, H)$. Clearly, $a$ goes to $x$, for $\prod_{p \mid N} \pi_{p}$ is an isomorphism.

Proof of reduction. We are going to show $\left(7_{p}\right) \Longleftrightarrow\left(6_{p}\right) \Longleftrightarrow\left(5_{p}\right) \Longleftrightarrow$ $\left(4_{p}\right) \Longleftrightarrow\left(3_{p}\right) \Longrightarrow\left(2_{p}\right)$.

$\left(7_{p}\right) \Longleftrightarrow\left(6_{p}\right)$ This follows from the topological isomorphism

$$
A\left(B F_{i}, B K\right)_{p}^{\wedge} \rightarrow\left\{B F_{i_{+}}, B K_{+}\right\}_{p}^{\wedge},
$$

proved in [MSZ].

$\left(6_{p}\right) \Longleftrightarrow\left(5_{p}\right)$ This is because $A(P, K) \rightarrow \underline{\lim }_{i}\left\{B F_{i_{+}}, B K_{+}\right\}_{p}^{\wedge}$ is the direct sum of $I(P, K) \rightarrow \lim _{n}\left\{B P^{(n)}, B K_{+}\right\}_{p}^{\wedge}$ and the canonical map $\mathbb{Z} \cong A(\{e\}, K) \rightarrow\left\{S^{0}, B K_{+}\right\}_{p}^{\wedge} \cong \mathbb{Z}_{p}^{\wedge}$

$\left(5_{p}\right) \Longleftrightarrow\left(4_{p}\right)$ For any spectrum $E$, we follow [Bo] to define its $p$-adic completion $E_{p}^{\wedge}$ to be the function spectrum $F\left(\Sigma^{-1} M\left(\mathbb{Z} / p^{\infty}\right), E\right)$, where $M(A)$ is the Moore spectrum of an abelian group $A$ s.t. $\pi_{0}(M(A))=A, \pi_{i}(M(A))=0$ if $i \neq 0$. Suppose $\pi_{i} E$ is finitely generated for any $i$, then $\pi_{*}\left(E_{p}^{\wedge}\right) \cong\left(\pi_{*} E\right)_{p}^{\wedge}$ (Proposition $2.5[\mathrm{Bo}])$. Notice that the $p$-adic topology of the right-hand side is induced from the filtration associated with the expression $\operatorname{holim}_{m} \Sigma^{-1} M\left(\mathbb{Z} / p^{m}\right)$ $=\Sigma^{-1} M\left(\mathbb{Z} / p^{\infty}\right)$ of the left-hand side.

Consider a spectrum $X=\operatorname{holim}_{n} X_{n}$ with each $X_{n}$ a finite spectrum. We equip $\left(E_{p}^{\wedge}\right)^{q}(X)$ with the induced topology with respect to the canonical isomorphism $\left(E_{p}^{\wedge}\right)^{q}(X)=\varliminf_{n}\left(E_{p}^{\wedge}\right)^{q}\left(X_{n}\right)$. Thus, using Proposition 2.5 [Bo] again, we

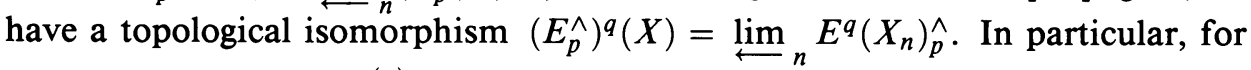
$X=B P$ and $X_{n}=B P^{(n)}$, we have

$$
\left(E_{p}^{\wedge}\right)^{q}(B P)=\underbrace{\lim }_{n} E^{q}\left(B P^{(n)}\right)_{p}^{\wedge} .
$$

But, as the canonical map $B F_{\infty} \cong \operatorname{holim}_{n} B F_{n}^{(n)} \rightarrow \operatorname{holim}_{n} B P^{(n)}=B P$ induces an isomorphism of mod- $p$ homology, we have the following sequence of topological isomorphisms:

$$
\begin{aligned}
& \left(E_{p}^{\wedge}\right)^{q}(B P)=\left(E_{p}^{\wedge}\right)^{q}\left(B F_{\infty}\right)=\underbrace{\lim }_{n}\left(E_{p}^{\wedge}\right)^{q}\left(B F_{n}^{(n)}\right) \\
& =\overleftarrow{i, n}_{i, n}^{\lim }\left(E_{p}^{\wedge}\right)^{q}\left(B F_{i}^{(n)}\right)=\underbrace{\lim }_{i}{\underset{n}{n}}_{\lim }\left(E_{p}^{\wedge}\right)^{q}\left(B F_{i}^{(n)}\right) \\
& =\underbrace{\lim }_{i} \underset{n}{\lim } E^{q}\left(B F_{i}^{(n)}\right)=\underbrace{\lim }_{i} E^{q}\left(B F_{i}\right) \text {, }
\end{aligned}
$$


where the topology of $E^{q}\left(B F_{i}\right)$ is the skeletal topology, and the fifth equality follows from the fact that the image of $E^{q}\left(B F_{i}^{(n)}\right) \rightarrow E^{q}\left(B F_{i}^{(n-1)}\right)$ is a finite $p$-group. Now, we specialize ourselves to the case $E=\Sigma^{\infty} B K_{+}$and $q=0$. Then the main theorem of [MSZ] implies that $\left\{B F_{i}, B K_{+}\right\}$is topologically isomorphic to the $p$-adic completion of the finitely generated free abelian group $I\left(F_{i}, K\right)$. In summary, we have the topological isomorphisms

$$
\underbrace{\lim _{n}}_{n}\left\{B P^{(n)}, B K_{+}\right\}_{p}^{\wedge} \cong\left\{B P,\left(B K_{+}\right)_{p}^{\wedge}\right\} \cong \underbrace{\lim }_{i}\left\{B F_{i}, B K_{+}\right\}_{p}^{\wedge},
$$

which clearly imply $\left(5_{p}\right) \Longleftrightarrow\left(4_{p}\right)$. (Compare with [F2].)

$\left(4_{p}\right) \Longleftrightarrow\left(3_{p}\right)$ This is the same as $\left(6_{p}\right) \Longleftrightarrow\left(5_{p}\right)$.

$\left(3_{p}\right) \Longrightarrow\left(2_{p}\right)$ Given a compact Lie group $G$, and its closed subgroups $H_{1}$ and $H_{2}$, we set $\xi\left(H_{1}, G\right): B H_{1+} \rightarrow B G_{+}$and $\tau\left(H_{2}, G\right): B G_{+} \rightarrow B H_{2+}$ to be the induced map of the inclusion $H_{1} \subset G$ and the stable transfer map associated with the fiber bundle $\mathrm{BH}_{2} \rightarrow B G$, respectively. Furthermore, let $\left[{ }_{H_{1}} G_{H_{2}}\right] \in A\left(H_{1}, H_{2}\right)$ be an element, which is represented by $G$ considered as a compact $H_{1} \times H_{2}$ manifold with respect to the left (free) $H_{1}$ and the right $\mathrm{H}_{2}$ actions given by the multiplication of $G$.

With these notations, Theorem 2.2 and Corollary 2.3 imply the following diagram commutes:

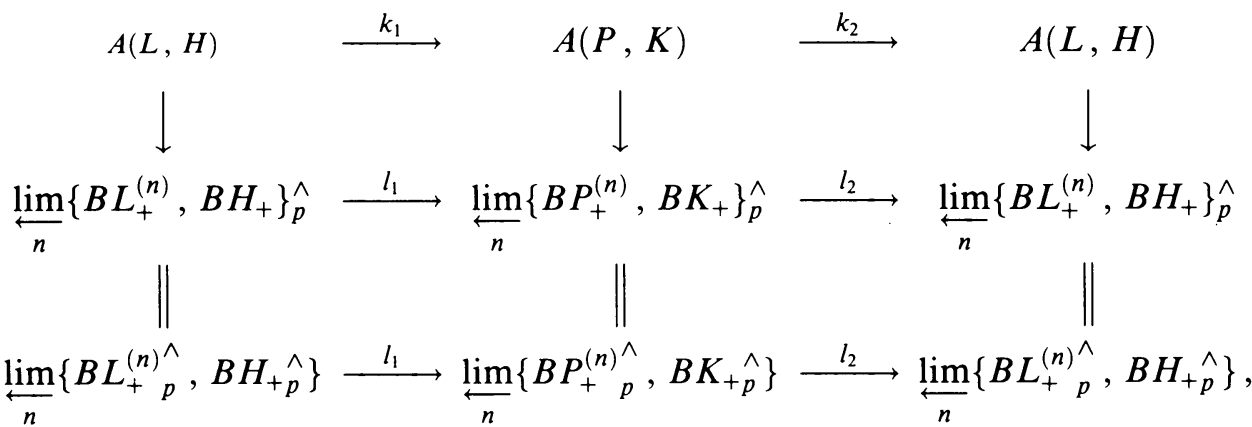

where

$$
\begin{aligned}
& k_{1}=\mathscr{C}\left(\left[{ }_{P} L_{L}\right] \otimes-\otimes\left[{ }_{H} H_{K}\right]\right), \quad k_{2}=\mathscr{C}\left(\left[{ }_{L} L_{P}\right] \otimes-\otimes\left[{ }_{K} H_{H}\right]\right), \\
& l_{1}=\tau(K, H) \circ-\circ \xi(P, L), \quad l_{2}=\xi(K, H) \circ-\circ \tau(P, L) .
\end{aligned}
$$

But both the composites

$$
\begin{aligned}
& \xi(P, L) \tau(P, L): B L_{+}^{(n)} \rightarrow B P_{+}^{(n)} \rightarrow B L_{+}^{(n)}, \\
& \xi(K, H) \tau(K, H): B H_{+} \rightarrow B K_{+} \rightarrow B H_{+},
\end{aligned}
$$

induce isomorphisms of the mod- $p$ homology, since both $\chi(L / P)$ and $\chi(H / K)$ are finite and relatively prime to $p$. This immediately implies that the composite $l_{2} \circ l_{1}$ is an isomorphism. Now, $\left(3_{p}\right) \Longrightarrow\left(2_{p}\right)$ follows immediately by the diagram chase in the above commutative diagram. This completes the proof of Reduction. 


\section{REFERENCES}

[AGM] J. F. Adams, J. H. Gunawardena, and H. R. Miller, The Segal conjecture for elementary p-groups, Topology 24 (1985), 435-460.

[B] S. Bauer, On the Segal conjecture for compact Lie groups, J. Reine Angew. Math. 400 (1989), 134-145.

[BG] J. Becker and D. Gottlieb, The transfer and fiber bundles, Topology 14 (1975), 1-13.

[Bo] A. K. Bousfield, The localization of spectra with respect to homology, Topology 18 (1979), 257-281.

[BK] A. K. Bousfield and D. M. Kan, Homotopy limits, completions and localizations, Lecture Notes in Math., vol. 304, Springer-Verlag, 1972.

[Br] G. E. Bredon, Introduction to compact transformation groups, Academic Press, 1972.

[C] G. Carlsson, Eequivariant stable homotopy and Segal's Burnside ring conjecture, Ann. of Math. 120 (1984), 189-224.

[D] T. tom Dieck, Transformation groups and representation theory, Lecture Notes in Math., vol. 766, Springer-Verlag, 1979.

[D2] - Transformation groups, De Gruyter, Berlin-NewYork, 1987.

[DP] T. tom Dieck and T. Petrie, Geometric modules over the Burnside ring, Invent. Math. 47 (1978), 273-287.

[Do1] A. Dold, Lectures on algebraic topology, Springer-Verlag, Berlin-Heidelberg-New York, 1972.

[Do2] _ The fixed point index of fibre-preserving maps, Invent. Math. (1974), 281-297.

[Do3] — The fixed point transfer of fibre-preserving maps, Math. Z. (1976), 281-297.

[F1] M. Feshbach, The transfer and compact Lie groups, Trans. Amer. Math. 251 (1979), 139169.

[F2] M. Feshbach, The Segal conjecture for compact Lie groups, Topology 26 (1987), 1-20.

[J] J. W. Jaworowski, Extensions of G-maps and Euclidean G-retracts, Math. Z. 146 (1976), 143-148.

[L] E. Laitinen, On the Burnside ring and stable cohomotopy of a finite group, Math. Scand. 44 (1979), 37-72.

[LM] R. K. Lashof and J. P. May, Generalized equivariant bundles, Bull. Soc. Math. Belgique 38 (1986), 265-271.

[LMSe] R. K. Lashof, J. P. May and G. B. Segal, Equivariant bundles with abelian structured group, Homotopy Theory, Proc. Conf., Evanston 1982; Contemporary Math., vol. 19, Amer. Math. Soc., Providence, RI, 1983, pp. 167-176.

[LM2] C.-N. Lee and N. Minami, Segal's Burnside ring conjecture for compact Lie groups, Algebraic Topology and Its Applications, Springer-Verlag, New York, 1994, pp. 133-161.

[LMM] L. G. Lewis, Jr., J. P. May and J. E. McClure, Classifying G-spaces and the Segal conjecture, Canadian Math. Soc. Conference Proceedings, Vol. 2, part 1, 1982, pp. 165-179.

[LMS] L. G. Lewis, Jr., J. P. May and M. Steinberger (with contributions by J. E. McClure), Equivariant stable homotopy theory, Lecture Notes in Math., vol. 1213, Springer-Verlag, Berlin-New York, 1986.

[MP] S. Mitchell and S. Priddy, A double coset formula for Levi subgroups and splitting $B G L_{n}$, Lecture Notes in Math., vol. 1370, Springer, 1989, pp. 325-334.

[MSZ] J. P. May, V. P. Snaith, and P. Zelewski, A further generalization of the Segal conjecture, Quart. J. Math. 40 (1989), 457-473.

[Ma] J. P. May, Some remarks on equivariant bundles and classifying spaces, preprint.

[M1] N. Minami, On the $I(G)$-adic topology of the Burnside rings of compact Lie groups, Publ. Res. Inst. Math. Sci. 20 (1984), 447-460.

[M2] Group homomorphisms inducing an isomorphism of a functor, Math. Proc. Cambridge Philos. Soc. 104 (1988), 81-93.

[N] G. Nishida, On the $S^{1}$-Segal conjecture, Publ. Res. Inst. Math. Sci. 19 (1983), 1153-1162. 
[P] S. Priddy, On characterizing summands in the classifying space of groups, II, Lecture Notes in Math., vol. 1418, Springer, 1990.

[R] I. Reiner, Maximal orders, Academic Press, London, 1975.

[S] J. P. Serre, Cohomologie Galoisienne, Lecture Notes in Math., vol. 5, Springer-Verlag, Berlin, 1964.

[S1] V. P. Snaith, Explicit Brauer induction, Invent. Math. 94 (1988), 455-478.

[S2] _ _ Topological methods in Galois representation theory, Wiley, New York, 1989.

Department of Mathematics, The University of Alabama, Box 870350, Tuscaloosa, Alabama 35487-0350

E-mail address: norihiko@gp.as.ua.edu, norihiko@euler.math.ua.edu 